\title{
Lattice approach to finite volume form-factors of the Massive Thirring (Sine-Gordon) model
}

\author{
Árpád Hegedüs \\ MTA Lendület Holographic QFT Group, Wigner Research Centre, \\ H-1525 Budapest 114, P.O.B. 49, Hungary \\ E-mail: hegedus.arpad@wigner.mta.hu
}

ABSTRACT: In this paper we demonstrate, that the light-cone lattice approach for the Massive-Thirring (sine-Gordon) model, through the quantum inverse scattering method, admits an appropriate framework for computing the finite volume form-factors of local operators of the model. In this work we compute the finite volume diagonal matrix elements of the $\mathrm{U}(1)$ conserved current in the pure soliton sector of the theory. Based on the systematic large volume expansion of our results, we conjecture an exact expression for the finite volume expectation values of local operators in pure soliton states. At large volume in leading order these expectation values have the same form as in purely elastic scattering theories, but exponentially small corrections differ from previous Thermodynamic Bethe Ansatz conjectures of purely elastic scattering theories.

KEywords: Bethe Ansatz, Integrable Field Theories, Lattice Integrable Models

ArXiv EPRINT: 1705.00319 


\section{Contents}

1 Introduction 1

2 Light-cone approach to the Massive-Thirring and sine-Gordon models 3

2.1 Algebraic Bethe ansatz 5

$\begin{array}{ll}2.2 & \text { The DDV equations }\end{array}$

2.3 The $\mathrm{U}(1)$ current in spin variables 9

3 Form-factors in the QISM framework $\quad 10$

4 The computation of $\left\langle e_{n}\right\rangle_{\lambda} \quad 13$

$\begin{array}{ll}4.1 \text { The determination of } S_{a} & 14\end{array}$

$\begin{array}{llr}5 & \text { The continuum limit } & \mathbf{1 7}\end{array}$

$\begin{array}{ll}5.1 \text { The solution of the equations } & 18\end{array}$

$\begin{array}{lll}5.1 .1 & \text { The charge density case } & 18\end{array}$

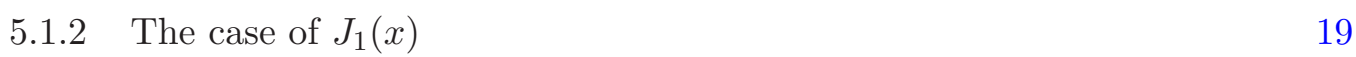

$\begin{array}{lll}6 & \text { The large volume expansion } & 20\end{array}$

$\begin{array}{lll}7 & \text { Summary and outlook } & 25\end{array}$

A Conventions of Fourier transformation $\quad 26$

\section{Introduction}

The computation of finite volume matrix elements of local operators is an important problem in integrable quantum field theories. These form-factors play important role in the determination of heavy-heavy-light 3-point functions in the planar $A d S_{5} / C F T_{4}$ correspondence [1], and they are fundamental ingredients of the form-factor perturbation theory [2].

In [3] the Massive Thirring (MT) model was formulated as the continuum limit of an inhomogeneous 6-vertex model with appropriately chosen alternating inhomogeneities. This integrable lattice regularization allowed one to compute the finite volume spectrum of the theory by solving a set of nonlinear-integral equations (NLIE) [4]-[11]. Due to the bosonization link between the Massive Thirring and sine-Gordon models [13, 14], this method gave access to the finite volume spectrum of the sine-Gordon (SG) model as well. The NLIE description of the finite volume spectrum was checked against direct field theoretical methods such as the Truncated Conformal Space Approach (TCSA) as well [10].

Nevertheless, the integrable lattice regularization of [3] gives access to compute matrix elements of local operators of the MT model and of their bosonized counterparts in the SG 
model. The general framework for these computations is the Quantum Inverse Scattering Method (QISM) [15]. In the past decades a remarkable amount of progress has been achieved in the computation of form-factors and correlation functions of local spin operators on the lattice [16]-[37]. One of the most important discovery was that local spin operators can be expressed in terms of the elements of the Yang-Baxter algebra in an elegant way [17]. This made it possible to compute the matrix elements of local spin operators by using only the Yang-Baxter algebra.

Relying on the light-cone lattice regularization of [3], in this paper our purpose is to compute finite volume form-factors of local operators in the MT/SG theories. The lattice Fermi fields of the regularized MT model are related to the spin operators by a Jordan-Wigner transformation. This is why the results of the QISM for spin variables are directly applicable to our model. Nevertheless, due to renormalization effects ${ }^{1}$ the connection between lattice fields and the fields of the continuum theory can be very nontrivial. Because of these subtleties in this paper we restrict ourselves to operators which are related to the U(1) symmetry of the model. Since the U(1) symmetry is present in both the lattice and the continuum theories, it makes easier to make a connection between the lattice and the continuum fields. The principle is that conserved quantities of the regularized theory are mapped to conserved quantities of the continuum model. In this manner we can identify the two components of the conserved $\mathrm{U}(1)$ current $^{2}$ of the continuum theory as $J_{0}\left(x_{n}\right) \sim \frac{\sigma_{2 n}^{z}+\sigma_{2 n-1}^{z}}{2}, \quad J_{1}\left(x_{n}\right) \sim \frac{\sigma_{2 n}^{z}-\sigma_{2 n-1}^{z}}{2}$.

Using the QISM techniques the diagonal matrix elements of $J_{\mu}$ can be computed on the lattice and the continuum limit can be taken as well. The final results can be expressed in terms of the counting-function of the theory, which satisfies a set of NLIEs [9]-[11], which we will refer to as DDV equations. For the sake of simplicity, in our actual computations we restricted ourselves to the pure soliton sector ${ }^{3}$ of the theory, but the computations could be extended without any serious difficulties to other excited states of the model, as well.

For $J_{0}$ we got the expected and quite trivial result, that the expectation value is equal to the topological charge of the state divided by the volume. For $J_{1}$ the result is not so trivial. There the expectation value can be expressed by the solution of a linear integral equation, whose kernel depend on the counting-function of the sandwiching state. These equations can be solved analytically in the context of a systematic large volume expansion.

It turns out, that in accordance with [43], in the pure soliton sector, at large volume in leading order the diagonal form-factors of $J_{\mu}$ can be expressed in terms of the socalled connected-form factors of the operator in exactly the same way as in purely elastic scattering theories [41, 42]. Nevertheless the exponentially small in volume corrections differ from the TBA conjectures [38-42] of purely elastic scattering theories. The difference arises in the form of the so-called dressed-form factors, which in our case are functionals of the counting-function of the sandwiching state and the connected-form factors of $J_{\mu}(6.26)$.

Based on previous experiences in diagonally scattering theories, we conjecture that in the pure soliton sector, our final formula (6.26) for the dressed form-factors hold for

\footnotetext{
${ }^{1}$ Here we think of normal ordering, renormalization constants and operator mixing.

${ }^{2}$ The corresponding conserved quantity is the toplogical charge in the SG model.

${ }^{3}$ In lattice terminology: we restrict ourselves to pure hole states over the antiferromagnetic vacuum.
} 
any operator, provided the connected form-factors of the operator under consideration are substituted into (6.26).

The organization of the paper is as follows. In section 2. we summarize the lightcone lattice approach to the MT model and determine the lattice counterparts of the U(1) conserved current. The NLIE governing the finite volume spectrum of the model is also reviewed in this section. In section 3. we provide the integrable QISM formulation of the model. In section 4. the diagonal matrix elements of the operator $\sigma_{n}^{z}$ are computed on the lattice. The continuum equations, their solutions and the correct identification between the lattice and continuum fields are presented in section 5. The systematic large volume expansion and the determination of dressed form factors can be found in section 6 . Our summary and outlook can be found in section 7 . The paper includes a short appendix containing some Fourier-transforms being necessary for the computations.

\section{Light-cone approach to the Massive-Thirring and sine-Gordon models}

The continuum models we consider in this paper are the sine-Gordon theory,

$$
\mathcal{L}_{\mathrm{SG}}=\frac{1}{2} \partial_{\nu} \Phi \partial^{\nu} \Phi+\frac{\mu^{2}}{\beta^{2}}: \cos (\beta \Phi): \quad 0<\beta^{2}<8 \pi,
$$

and the massive Thirring model:

$$
\mathcal{L}_{\mathrm{MT}}=\bar{\Psi}\left(i \gamma_{\nu} \partial^{\nu}+m_{0}\right) \Psi-\frac{g}{2} \bar{\Psi} \gamma^{\nu} \Psi \bar{\Psi} \gamma_{\nu} \Psi
$$

where we use chiral representation for the fermions $\left\{\gamma^{\mu}, \gamma^{\nu}\right\}=2 \eta^{\mu \nu}$ :

$$
\Psi=\left(\begin{array}{l}
\psi_{L} \\
\psi_{R}
\end{array}\right), \quad \gamma^{0}=\left(\begin{array}{ll}
0 & 1 \\
1 & 0
\end{array}\right), \quad \gamma^{1}=\left(\begin{array}{cc}
0 & 1 \\
-1 & 0
\end{array}\right), \quad \gamma^{5}=\gamma^{0} \gamma^{1}=-\eta=\left(\begin{array}{cc}
-1 & 0 \\
0 & 1
\end{array}\right) .
$$

By bosonization techniques, it was shown [13] that the two models can be mapped into each other provided their coupling constants satisfy the relation:

$$
1+\frac{g}{4 \pi}=\frac{4 \pi}{\beta^{2}} .
$$

There is a subtle point in the equivalence of the two theories [14], namely they are equivalent only in the even topological charge sector of their Hilbert-spaces and they differ in the odd topological charge sector.

The light-cone lattice approach of [3] provides an integrable lattice regularization of the MT model in the even topological charge sector of theory. In this description the space-time is discretized along the light-cone directions: $x_{ \pm}=x \pm t$ with an even number of lattice sites in the spatial direction. The sites of the light-cone lattice correspond to the discretized points of space-time. The left- and right-mover fermion fields live on the leftand right-oriented edges of the lattice. In this manner a left- and a right-mover fermion field can be assigned to each site of the lattice. (See figure 1.)

Lattice fermion fields satisfy the anticommutation relations:

$$
\left\{\psi_{A, n}, \psi_{B, m}\right\}=0, \quad\left\{\psi_{A, n}, \psi_{B, m}^{+}\right\}=\delta_{A B} \delta_{n m}, \quad A, B=R, L, \quad 1 \leq m, n \leq N .
$$




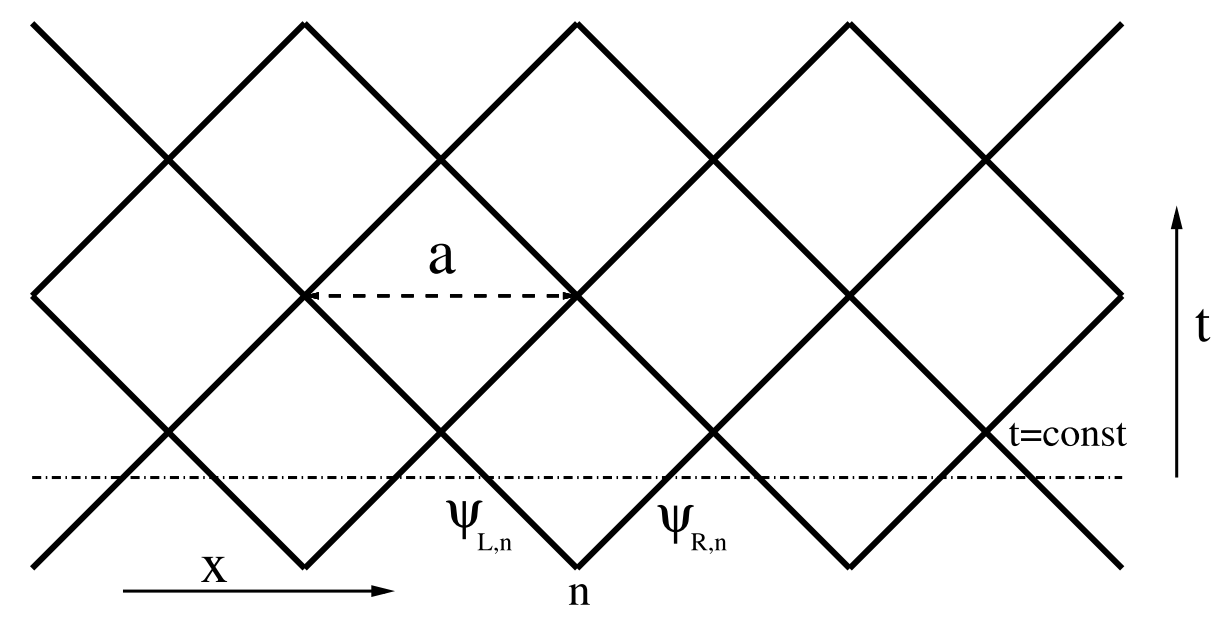

Figure 1. The pictorial representation of the light-cone lattice.

Then left- and right-mover fields live on the odd and even edges of of the light-cone lattice respectively:

$$
\psi_{R, n}=\psi_{2 n}, \quad \psi_{L, n}=\psi_{2 n-1}, \quad 1 \leq n \leq \frac{N}{2} .
$$

In this regularization, the variables $\psi_{n}$ are used to formulate the model and they are related to the commonly used spin variables by a Jordan-Wigner transformation:

$$
\psi_{n}^{+}=\sigma_{n}^{+} \prod_{l=1}^{n-1} \sigma_{l}^{z}, \quad \psi_{n}=\sigma_{n}^{-} \prod_{l=1}^{n-1} \sigma_{l}^{z} .
$$

The $U_{L}$ and $U_{R}$ light-cone evaluation operators of the model are given by inhomogeneous transfer matrices of the 6 -vertex model with appropriate alternating inhomogeneities as follows.

Let us consider the 6 -vertex model with the following $R$-matrix:

$$
R(\lambda)=\left(\begin{array}{cccc}
1 & 0 & 0 & 0 \\
0 & \frac{\sinh (\lambda)}{\sinh (\lambda-i \gamma)} & \frac{\sinh (-i \gamma)}{\sinh (\lambda-i \gamma)} & 0 \\
0 & \frac{\sinh (-i \gamma)}{\sinh (\lambda-i \gamma)} & \frac{\sinh (\lambda)}{\sinh (\lambda-i \gamma)} & 0 \\
0 & 0 & 0 & 1
\end{array}\right),
$$

where $\lambda$ is the spectral parameter and $\gamma$ is the anisotropy parameter which encodes the coupling dependence of the MT model. The coupling dependence of $\gamma$ is given by:

$$
\gamma=\frac{\pi}{p+1}, \quad 0<p<\infty
$$

where $p$ parameterizes the coupling constant of the SG and MT models by the formula: ${ }^{4}$

$$
\frac{\beta^{2}}{4 \pi}=\frac{1}{1+\frac{g}{4 \pi}}=\frac{2 p}{p+1} .
$$

\footnotetext{
${ }^{4}$ This parameterization is introduced to relate our results easier to the DDV equation.
} 
The R-matrix (2.7) acts on the tensor product of two linear spaces both being isomorfic to $\mathbb{C}^{2}$. As usual, the $R$-matrix acting on $V_{1}\left(\lambda_{1}\right) \otimes V_{2}\left(\lambda_{2}\right)$ is denoted by $R_{12}\left(\lambda_{1}-\lambda_{2}\right)$. The monodromy matrix acts on $V_{0}$ and the quantum space of the model $\mathcal{H}=\otimes_{i=1}^{N} V_{i}$ and is given by:

$$
T(\lambda \mid \vec{\xi})=R_{01}\left(\lambda-\xi_{1}\right) R_{02}\left(\lambda-\xi_{2}\right) \ldots R_{0 N}\left(\lambda-\xi_{N}\right)=\left(\begin{array}{ll}
A(\lambda) & B(\lambda) \\
C(\lambda) & D(\lambda)
\end{array}\right)_{[0]}
$$

where $\vec{\xi}$ is the $N$-dimensional inhomogeneity vector given by:

$$
\vec{\xi}=\left\{\xi_{-}, \xi_{+}, \xi_{-}, \xi_{+}, \ldots, \xi_{-}, \xi_{+}\right\}
$$

with

$$
\xi_{ \pm}= \pm \rho-i \frac{\gamma}{2}
$$

Here the parameter $\rho$ is part of the regularization scheme. This is why it depends on the lattice spacing or equivalently on the number of lattice sites. This dependence is given by the formula:

$$
\rho=\frac{\gamma}{\pi} \ln \frac{4}{\mathcal{M} a}=\frac{\gamma}{\pi} \ln \frac{2 N}{\mathcal{M} L},
$$

where $\mathcal{M}$ is the physical mass of fermions (solitons), $a$ denotes the lattice spacing, $N$ is the number ${ }^{5}$ of lattice sites of the 6 -vertex model and $L$ is the volume. Due to the integrability of the model the transfer matrixes form a commutative family of operators on the quantum space of the model:

$$
\mathcal{T}(\lambda \mid \vec{\xi})=\operatorname{Tr}_{0} T(\lambda \mid \vec{\xi}), \quad\left[\mathcal{T}(\lambda \mid \vec{\xi}), \mathcal{T}\left(\lambda^{\prime} \mid \vec{\xi}\right)\right]=0
$$

The $U_{L}$ and $U_{R}$ light-cone evaluation operators of the regularized MT model are given by the transfer matrices:

$$
U_{L}=e^{i \frac{2}{a}(H-P)}=\mathcal{T}\left(\xi_{+} \mid \vec{\xi}\right), \quad U_{R}^{+}=e^{-i \frac{2}{a}(H+P)}=\mathcal{T}\left(\xi_{-} \mid \vec{\xi}\right),
$$

where $H$ is the Hamiltonian and $P$ is the momentum of the model. From this description it follows that the eigenstates of the Hamiltonian are the eigenvectors of the commuting transfer matrices. These eigenvectors can be obtained via the algebraic Bethe Ansatz technique [15].

\subsection{Algebraic Bethe ansatz}

In the framework of algebraic Bethe Ansatz method, the eigenstates of the mutually commuting family of transfer matrices (2.14) are constructed by acting with a product of $B$-operators on the reference state $|0\rangle$, which is the completely ferromagnetic $S_{z}=\frac{N}{2}$ state of the model:

$$
|\vec{\lambda}\rangle=\left|\lambda_{1}, \lambda_{2}, . ., \lambda_{m}\right\rangle=B\left(\lambda_{1}\right) B\left(\lambda_{2}\right) \ldots B\left(\lambda_{m}\right)|0\rangle, \quad S_{z}|\vec{\lambda}\rangle=\left(\frac{N}{2}-m\right)|\vec{\lambda}\rangle .
$$

\footnotetext{
${ }^{5}$ In this convention, in the light-cone lattice the number of lattice sites in spatial direction is $\frac{N}{2}$. See figure 1.
} 
Such a state is an eigenstate provided the spectral parameters in the argument of the $B$-operators satisfy the Bethe equations:

$$
\prod_{i=1}^{N} \frac{\sinh \left(\lambda_{a}-\xi_{i}-i \gamma\right)}{\sinh \left(\lambda_{a}-\xi_{i}\right)} \prod_{b=1}^{m} \frac{\sinh \left(\lambda_{a}-\lambda_{b}+i \gamma\right)}{\sinh \left(\lambda_{a}-\lambda_{b}-i \gamma\right)}=-1, \quad a=1, \ldots, m
$$

The eigenvalues of the transfer matrices can also be expressed in terms of the Bethe-roots:

$$
\mathcal{T}_{\vec{\lambda}}(\mu \mid \vec{\xi})=\prod_{k=1}^{m} \frac{\sinh \left(\mu-\lambda_{k}+i \gamma\right)}{\sinh \left(\mu-\lambda_{k}\right)}+\prod_{i=1}^{N} \frac{\sinh \left(\mu-\xi_{i}\right)}{\sinh \left(\mu-\xi_{i}-i \gamma\right)} \prod_{k=1}^{m} \frac{\sinh \left(\mu-\lambda_{k}-i \gamma\right)}{\sinh \left(\mu-\lambda_{k}\right)}
$$

The Bethe-equations can be reformulated in terms of the so-called counting-function $Z_{\lambda}(\lambda)$ :

$$
(-1)^{\delta} e^{i Z_{\lambda}\left(\lambda_{a}\right)}=-1, \quad \delta=m(\bmod 2), \quad a=1, . ., m,
$$

where

$$
(-1)^{\delta} e^{i Z_{\lambda}(\lambda)}=\prod_{i=1}^{N} \frac{\sinh \left(\lambda-\xi_{i}-i \gamma\right)}{\sinh \left(\lambda-\xi_{i}\right)} \prod_{b=1}^{m} \frac{\sinh \left(\lambda-\lambda_{b}+i \gamma\right)}{\sinh \left(\lambda-\lambda_{b}-i \gamma\right)}
$$

For the proper definition of $Z_{\lambda}(\lambda)$ the logarithm of (2.20) should be taken, such that the counting function should be continuous along the real axis. This can be achieved by defining the function [8]:

$$
\phi_{\nu}(\lambda)=-i \log \frac{\sinh \left(i \frac{\gamma}{2} \nu-\lambda\right)}{\sinh \left(i \frac{\gamma}{2} \nu+\lambda\right)}, \quad 0<\nu, \quad \phi_{\nu}(0)=0, \quad|\operatorname{Im} \lambda|<\nu .
$$

The function $\phi_{\nu}(\lambda)$ can be continued analytically to the regime $|\operatorname{Im} \lambda|>\nu$ by the requirements that its logarithmic discontinuities should run parallel to the real axis and it should be an odd function on the entire complex plane. Using this analytically continued $\phi_{\nu}(\lambda)$, the definition of the counting-function specified to the inhomogeneities (2.12) is given by the formula [8]:

$$
Z_{\lambda}(\lambda)=\frac{N}{2}\left(\phi_{1}(\lambda-\rho)+\phi_{1}(\lambda+\rho)\right)-\sum_{k=1}^{m} \phi_{2}\left(\lambda-\lambda_{k}\right) .
$$

Using $Z_{\lambda}(\lambda)$, the Bethe-equations (2.17) can be reformulated in their logarithmic form by the formula:

$$
Z_{\lambda}\left(\lambda_{a}\right)=2 \pi I_{a}, \quad I_{a} \in \mathbb{Z}+\frac{1+\delta}{2} \quad a=1, . ., m .
$$

We note that the role of $\delta$ is to determine whether the quantum numbers $I_{a}$ should be integers or half-integers. The vacuum of the field theory corresponds to the $\delta=0, S_{z}=0$, antiferromagnetic vacuum of the lattice-model. ${ }^{6}$ This state is formed by $N / 2$ real Bethe-roots, such that to all quantum numbers satisfying the inequality $Z_{\lambda}(-\infty) \leq 2 \pi I_{a} \leq Z_{\lambda}(\infty)$ there exist a real Bethe-root in (2.23). The excitations above this vacuum are characterized by complex Bethe-roots and holes, where holes are such real solutions of (2.19), which are not Bethe-roots. In the logarithmic form of the equations quantum numbers can be assigned to holes as well:

$$
Z_{\lambda}\left(h_{k}\right)=2 \pi I_{k}, \quad I_{k} \in \mathbb{Z}+\frac{1+\delta}{2} \quad k=1, . ., m_{H},
$$

where $h_{k}$ denotes the positions of the holes and their number is denoted by $m_{H}$.

\footnotetext{
${ }^{6}$ According to (2.19), the $\delta=0$ requirement implies that $\frac{N}{2}$ must be even on the lattice.
} 


\subsection{The DDV equations}

The DDV equations ${ }^{7}$ [4]-[11] reformulate the Bethe-equations (2.17) in terms of a set of nonlinear-integral equations, such that only those objects enter the equations, which characterize the excitations. In this paper we will compute diagonal form factors in the pure soliton sector of the theory, thus we recall here the form of the DDV equation only for the pure soliton- or equivalently for pure hole states. The DDV equation for this sector was derived first in [7]. Here we present the equations in rapidity variables i.e. $\theta=\frac{\pi}{\gamma} \lambda$, because of two reasons. First, this way it is easier to find connection to the literature of the DDV equation [5]-[11], and on the other hand at the stage of our final results it is better to work in this convention, since in the field theory this variable corresponds to the rapidity of particles. We recall the DDV equation for both the lattice and for the continuum theories. To do so, first we relate the lattice counting function in rapidity variables to $Z_{\lambda}(\lambda)$ of $(2.22)$. The relation is given by $Z_{N}(\theta)=Z_{\lambda}\left(\frac{\gamma}{\pi} \theta\right)$. The DDV equation for $Z_{N}(\theta)$ in the pure hole sector reads as:

$$
\begin{aligned}
Z_{N}(\theta)= & \frac{N}{2}\{\arctan [\sinh (\theta-\Theta)]+\arctan [\sinh (\theta+\Theta)]\}+\sum_{k=1}^{m_{H}} \chi\left(\theta-H_{k}\right) \\
& +\int_{-\infty}^{\infty} \frac{d \theta^{\prime}}{2 \pi i} G\left(\theta-\theta^{\prime}-i \eta\right) L_{N}^{(+)}\left(\theta^{\prime}+i \eta\right)-\int_{-\infty}^{\infty} \frac{d \theta^{\prime}}{2 \pi i} G\left(\theta-\theta^{\prime}+i \eta\right) L_{N}^{(-)}\left(\theta^{\prime}-i \eta\right),
\end{aligned}
$$

where $\chi(\theta)$ is the soliton-soliton scattering phase and $G(\theta)$ is its derivative:

$$
G(\theta)=-i \frac{d}{d \theta} \log S_{++}^{++}(\theta)=\int_{-\infty}^{\infty} d \omega e^{-i \omega \theta} \frac{\sinh \left(\frac{(p-1) \pi \omega}{2}\right)}{2 \cosh \left(\frac{\pi \omega}{2}\right) \sinh \left(\frac{p \pi \omega}{2}\right)},
$$

$0<\eta<\min (p \pi, \pi)$ is an arbitrary positive contour-integral parameter, which must be smaller than the distance of the first pole of $G(\theta)$ from the real axis. Furthermore, $L_{N}^{( \pm)}(\theta)$ denotes the nonlinear combinations of $Z_{N}(\theta)$ :

$$
L_{N}^{( \pm)}(\theta)=\ln \left(1+(-1)^{\delta} e^{ \pm i Z_{N}(\theta)}\right)
$$

$\Theta=\ln \frac{2 N}{\mathcal{M} L}$ is the inhomogeneity parameter and $H_{k}=\frac{\pi}{\gamma} h_{k}$ denote the positions of the holes in the rapidity convention. They are subjected to the quantization equations:

$$
Z_{N}\left(H_{k}\right)=2 \pi I_{k} \quad I_{k} \in \mathbb{Z}+\frac{1+\delta}{2} \quad k=1, . ., m_{H} .
$$

A counting-equation [8] can be derived, which tells us how the number of excitation characterizing objects is related to the spin or equivalently to the conserved quantum number of the state. For pure hole states without special objects ${ }^{8}$ the counting-equation on the lattice takes the form:

$$
m_{H}=2 S_{z}-2\left[\frac{1}{2}+\frac{S_{z}}{p+1}\right]
$$

\footnotetext{
${ }^{7} \mathrm{~A}$ detailed review on the DDV equations can be found in [12].

${ }^{8}$ Special objects are points on the complex plane, where the $L_{N}^{( \pm)}(\theta)$ jumps along the integration contour due to going though the branch cut of the logarithm. For more detail see for example $[8,12]$.
} 
where here [...] stands for integer part. Since $S_{z}=\frac{N}{2}-m$, this equation tells us that, on a lattice with even number of sites, only states with even number of holes exist. The lattice counting-function $Z_{N}(\theta)$ depends on the number of lattice sites $N$. It has a continuum limit, which is just its $N \rightarrow \infty$ limit $[5,6]$ :

$$
Z(\theta)=\lim _{N \rightarrow \infty} Z_{N}(\theta), \quad L_{ \pm}(\theta)=\lim _{N \rightarrow \infty} L_{N}^{( \pm)}(\theta)=\ln \left(1+(-1)^{\delta} e^{ \pm i Z(\theta)}\right) .
$$

With these notations the continuum DDV equations are just the $N \rightarrow \infty$ limit of the lattice ones (2.25):

$$
\begin{aligned}
Z(\theta)= & \ell \sinh \theta+\sum_{k=1}^{m_{H}} \chi\left(\theta-H_{k}\right)+\int_{-\infty}^{\infty} \frac{d \theta^{\prime}}{2 \pi i} G\left(\theta-\theta^{\prime}-i \eta\right) L_{+}\left(\theta^{\prime}+i \eta\right) \\
& -\int_{-\infty}^{\infty} \frac{d \theta^{\prime}}{2 \pi i} G\left(\theta-\theta^{\prime}+i \eta\right) L_{-}\left(\theta^{\prime}-i \eta\right)
\end{aligned}
$$

where $\ell=\mathcal{M} L$ with $L$ being the volume and $\mathcal{M}$ is the soliton mass. The energy and momentum of these hole states in the continuum read as:

$$
\begin{aligned}
& E=\mathcal{M} \sum_{k=1}^{m_{H}} \cosh H_{k}-\frac{\mathcal{M}}{2 \pi i} \sum_{\alpha= \pm} \alpha \cdot \int_{-\infty}^{\infty} d \theta \sinh (\theta+i \alpha \eta) L_{\alpha}(\theta+i \alpha \eta) \\
& P=\mathcal{M} \sum_{k=1}^{m_{H}} \sinh H_{k}-\frac{\mathcal{M}}{2 \pi i} \sum_{\alpha= \pm} \alpha \cdot \int_{-\infty}^{\infty} d \theta \cosh (\theta+i \alpha \eta) L_{\alpha}(\theta+i \alpha \eta)
\end{aligned}
$$

Since in the large volume limit $L_{\alpha}(\theta+i \alpha \eta) \rightarrow 0$, from (2.32) and (2.33) it can be seen that in the large volume limit the holes correspond to the rapidities of the solitons. This is why in the sequel we will refer to holes as solitons. It also turns out [8] that the counting equation (2.29) changes in the continuum and it reads: ${ }^{9}$

$$
Q=m_{H}
$$

where $Q$ is the $\mathrm{U}(1)$ (topological) charge of the continuum model.

The choice ${ }^{10}$ of $\delta$ is crucial in the continuum theory. In the even charge sector of the theory $\delta=0$. In the odd charge sector the choice $\delta=0$ corresponds to the MT fermions, while the $\delta=1$ choice describes the SG solitons [9]-[11].

Though in this paper we will make computations only in the twistless case, which can describe only the even topological charge sector of the model, we note that in [46] it has been shown that the odd charge sector can also be investigated from the lattice, if the 6 -vertex model with an twist angle $\omega=\frac{\pi}{2}$ is considered.

\footnotetext{
${ }^{9}$ For pure soliton states without special objects.

${ }^{10}$ On the lattice the actual value of $\delta$ can be influenced by the parity of $\frac{N}{2}$.
} 


\subsection{The U(1) current in spin variables}

Our purpose is to compute the finite volume form-factors of local operators of the MT/SG models in the framework of QISM. To achieve this plan, the first step is to relate the lattice operators to the continuum ones. Due to renormalization effects, this is a complicated task in general. To avoid complications ${ }^{11}$ coming from renormalization effects we will restrict our attention to operators related to the $\mathrm{U}(1)$ symmetry of the model. The $\mathrm{U}(1)$ symmetry is present in both the lattice and the continuum theories, thus it is plausible to assume that the $\mathrm{U}(1)$ conserved charge of the lattice theory is mapped to the $\mathrm{U}(1)$ charge of the continuum theory.

The counting equations (2.29) and (2.34) suggest ${ }^{12}$ the $Q \sim 2 S_{z}$ identification between the lattice and continuum conserved quantities. This helps us to define the correct normal ordering for the lattice fermion fields as follows. Assuming the $Q \sim 2 S_{z}$ relation, the lattice topological charge can be expressed by lattice fermion fields using a Jordan-Wigner transformation (2.6):

$$
Q \sim 2 S_{z}=\sum_{n=1}^{N} \sigma_{n}^{z}=\sum_{n=1}^{N}\left(\psi_{n}^{+} \psi_{n}-\frac{1}{2}\right) .
$$

In the continuum theory the topological charge is given by the integral:

$$
Q=\int_{0}^{L} d x\left(: \Psi_{R}^{+} \Psi_{R}:(x)+: \Psi_{L}^{+} \Psi_{L}:(x)\right)
$$

which can be approximated on the lattice by the discrete sum:

$$
Q \approx \sum_{n=1}^{\frac{N}{2}}\left(: \psi_{2 n}^{+} \psi_{2 n}:+: \psi_{2 n-1}^{+} \psi_{2 n-1}:\right)
$$

The comparison of (2.35) and (2.37) offers a natural definition for the normal ordering of lattice Fermi-fields:

$$
: \psi_{n}^{+} \psi_{n}:=\psi_{n}^{+} \psi_{n}-\frac{1}{2}=\sigma_{n}^{z} .
$$

Our purpose is to determine the lattice counterparts of the conserved current belonging to the U(1) current of the MT model. The index- 0 component of the current is the charge density. This can be computed from (2.35) and (2.38):

$$
Q \sim 2 S_{z}=\sum_{n=1}^{\frac{N}{2}}\left(\sigma_{2 n}^{z}+\sigma_{2 n-1}^{z}\right)=\sum_{n=1}^{\frac{N}{2}} a \frac{\sigma_{2 n}^{z}+\sigma_{2 n-1}^{z}}{a}=\sum_{n=1}^{\frac{N}{2}} a \cdot j_{0}(n a) \rightarrow \int_{0}^{L} d x j_{0}(x),
$$

where $a=\frac{2 L}{N}$ is the lattice constant and the index- 0 component of the current at the lattice sites can be expressed in terms of lattice spin variables as:

$$
j_{0}(n a)=\frac{\sigma_{2 n}^{z}+\sigma_{2 n-1}^{z}}{a}=\frac{N}{L} \frac{\sigma_{2 n}^{z}+\sigma_{2 n-1}^{z}}{2} .
$$

\footnotetext{
${ }^{11}$ Here we mostly think of operator mixing.

${ }^{12}$ At least for large enough values of $p$, when the integer part becomes zero.
} 
In the continuum theory the conserved current is given by:

$$
J_{\mu}=: \bar{\Psi} \gamma_{\mu} \Psi:, \quad \mu=0,1
$$

which can be written in component fields as:

$$
\begin{aligned}
& J_{0}=: \Psi_{R}^{+} \Psi_{R}:+: \Psi_{L}^{+} \Psi_{L}: \\
& J_{1}=: \Psi_{R}^{+} \Psi_{R}:-: \Psi_{L}^{+} \Psi_{L}:
\end{aligned}
$$

Since left- and right-mover fields live on the odd and even links of our lattice respectively, comparing (2.40) and (2.42) gives immediately the index-1 component of the current in terms of spin variables:

$$
j_{1}(n a)=\frac{\sigma_{2 n}^{z}-\sigma_{2 n-1}^{z}}{a}=\frac{N}{L} \frac{\sigma_{2 n}^{z}-\sigma_{2 n-1}^{z}}{2} .
$$

Consequently (2.40) and (2.43) indicates that the computation of form factors of the current $J_{\mu}$ is reduced to compute form factors of $\sigma_{n}^{z}$ on the lattice. This can be achieved within the framework of QISM [17, 18].

We close this section with an important remark concerning the continuum limit and our notations. It can be recognized, that as far as the notation is concerned, we made difference between the continuum and the lattice notations of the U(1) current. Namely, $J_{\mu}(x)$ denotes the current in the continuum field theory, while $j_{\mu}(x)$ denotes the lattice analog of the continuum current, the derivation of which was based on the identification of the topological charge of the continuum field theory with $2 S_{z}$ of the corresponding lattice theory. However, in section 5.1. it will turn out that the two quantities are not equal, but only proportional. We just anticipate their relation, which is given by the formula (5.10):

$$
J_{\mu}(x)=\frac{p}{p+1} j_{\mu}(x), \quad \mu=0,1 .
$$

We note, that the renormalization factor $\frac{p}{p+1}$ tends to 1 , as $p$ tends to infinity in accordance with the indication of (2.29).

\section{Form-factors in the QISM framework}

In the previous section we argued that the computation of form-factors of the U(1) current of the MT/SG model is equivalent to the determination of the form-factors of $\sigma_{n}^{z}$ on the lattice. Our approach to compute the finite volume form-factors of local operators having lattice counterparts, consists of two steps; first one should compute the form-factors on the lattice. The result will depend on the number of lattice sites $N$. Then the $N \rightarrow \infty$ limit of the lattice result gives the required result for the continuum theory. ${ }^{13}$ We will demonstarate, that this method works fine by the computation of the diagonal matrix elements of the U(1) current. The details of the computations enlight, that the diagonal matrix elements of other combinations of local Fermi fields and their derivatives can also

\footnotetext{
${ }^{13}$ In many cases the careful analysis of renormalization constants is also necessary.
} 
be computed by this method. Moreover, this procedure gives a theoretical framework also for the computation of nondiagonal matrix elements of the operators.

In this section we collect the most important formulas being necessary for the computations. Consider the following vector of the Hilbert-space:

$$
|\vec{\lambda}\rangle=B\left(\lambda_{1}\right) B\left(\lambda_{2}\right) \ldots B\left(\lambda_{m}\right)|0\rangle .
$$

This is called Bethe-state if the numbers $\lambda_{j}$ are arbitrary and is called Bethe-eigenstate if all $\lambda_{j}$ are solutions of the Bethe equations (2.17). Then the corresponding "bra" vector is given by:

$$
\langle\vec{\lambda}|=\langle 0| C\left(\lambda_{m}\right) \ldots C\left(\lambda_{2}\right) C\left(\lambda_{1}\right) .
$$

To get all form-factors of the current, one should be able to compute the lattice form factors:

$$
\frac{\left\langle\vec{\mu}\left|\sigma_{n}^{z}\right| \vec{\lambda}\right\rangle}{\sqrt{\langle\vec{\lambda} \mid \vec{\lambda}\rangle\langle\vec{\mu} \mid \vec{\mu}\rangle}},
$$

where both $|\vec{\mu}\rangle$ and $|\vec{\lambda}\rangle$ are Bethe-eigenstates, but in this paper we will focus on computing only the diagonal matrix elements:

$$
\left\langle\sigma_{n}^{z}\right\rangle_{\lambda}=\frac{\left\langle\vec{\lambda}\left|\sigma_{n}^{z}\right| \vec{\lambda}\right\rangle}{\langle\vec{\lambda} \mid \vec{\lambda}\rangle}
$$

which turns out to be a much simpler problem.

Here we recall the most important formulas [18], which are necessary to perform our calculations. In the computations only the Yang-Baxter algebra and the elements of the monodromy matrix (2.10) are used. This is why it is important to express the local spin operators in terms of the $A, B, C, D$ operators of the monodromy matrix (2.10). It has been done in [17] and the relations are summarized by the formula:

$$
E_{n}^{a b}=\prod_{i=1}^{n-1}(A+D)\left(\xi_{i}\right) T_{a b}\left(\xi_{n}\right) \prod_{i=n+1}^{N}(A+D)\left(\xi_{i}\right), \quad a, b=1,2,
$$

where the operator $E_{n}$ is given in terms of spin operators as follows:

$$
E_{n}^{11}=\frac{1}{2}\left(1_{n}+\sigma_{n}^{z}\right), \quad E_{n}^{12}=\sigma_{n}^{-}, \quad E_{n}^{21}=\sigma_{n}^{+}, \quad E_{n}^{22}=\frac{1}{2}\left(1_{n}-\sigma_{n}^{z}\right) .
$$

In our actual computations we use the 22-component of (3.5):

$$
e_{n}=\frac{1}{2}\left(1_{n}-\sigma_{n}^{z}\right)=\prod_{i=1}^{n-1}(A+D)\left(\xi_{i}\right) D\left(\xi_{n}\right) \prod_{i=n+1}^{N}(A+D)\left(\xi_{i}\right),
$$

where for short we introduced the notation $e_{n}=\frac{1}{2}\left(1_{n}-\sigma_{n}^{z}\right)$. We compute the expectation values of $e_{n}$ on the lattice, since apart from a trivial constant and sign it is equal to the required matrix element $\frac{1}{2}\left\langle\sigma_{n}^{z}\right\rangle_{\lambda}$. We note, that the lattice part of our computations is a special case of the computations done in [18] for the emptiness formation probability. This is why we will mostly use the logic and formulas of [18]. 
To compute $\left\langle e_{n}\right\rangle_{\lambda}$ from (3.7), one should know how the operator $D\left(\xi_{n}\right) \operatorname{acts}^{14}$ on the "bra"-vector (3.2). This is given by the following formula [18]:

$$
\langle 0| \prod_{k=1}^{m} C\left(\lambda_{k}\right) D\left(\xi_{n}\right)=\sum_{a=1}^{m} \frac{1}{r\left(\lambda_{a}\right)} \frac{\prod_{k=1}^{m} \sinh \left(\lambda_{a}-\lambda_{k}-i \gamma\right)}{\sinh \left(\lambda_{a}-\xi_{n}\right) \prod_{\substack{k=1 \\ k \neq a}}^{m} \sinh \left(\lambda_{a}-\lambda_{k}\right)}\langle 0| \prod_{\substack{k=1 \\ k \neq a}}^{m} C\left(\lambda_{k}\right) C\left(\xi_{n}\right),
$$

where we explicitely exploited that $\xi_{n}$ is one of the inhomogeneities of the vertex model and introduced:

$$
r(\lambda)=\prod_{j=1}^{N} \frac{\sinh \left(\lambda-\xi_{j}-i \gamma\right)}{\sinh \left(\lambda-\xi_{j}\right)} .
$$

As a consequence of (3.9) and (2.17) it satisfies the identities:

$$
\prod_{k=1}^{m} r\left(\lambda_{k}\right)=1, \quad \frac{1}{r\left(\xi_{j}\right)}=0, \quad j=1, \ldots, N .
$$

The last ingredient necessary for the computations is the scalar product of a Bethe-state and a Bethe-eingenstate. Let $|\mu\rangle$ an arbitrary Bethe-state in the sense of (3.1) and $|\lambda\rangle$ be a Bethe-eigenstate. Then their scalar product is given by the formula [36]:

$$
\langle\vec{\mu} \mid \vec{\lambda}\rangle=\langle\vec{\lambda} \mid \vec{\mu}\rangle=\prod_{l=1}^{N} \frac{1}{r\left(\mu_{l}\right)} \cdot \frac{\operatorname{det} H(\vec{\mu} \mid \vec{\lambda})}{\prod_{j>k} \sinh \left(\mu_{k}-\mu_{j}\right) \sinh \left(\lambda_{j}-\lambda_{k}\right)},
$$

where $H(\vec{\mu} \mid \vec{\lambda})$ is an $m \times m$ matrix with entries:

$$
H_{a b}(\vec{\mu} \mid \vec{\lambda})=\frac{\sinh (-i \gamma)}{\sinh \left(\lambda_{a}-\mu_{b}\right)}\left(r\left(\mu_{b}\right) \frac{\prod_{k=1}^{m} \sinh \left(\lambda_{k}-\mu_{b}-i \gamma\right)}{\sinh \left(\lambda_{a}-\mu_{b}-i \gamma\right)}-\frac{\prod_{k=1}^{m} \sinh \left(\lambda_{k}-\mu_{b}+i \gamma\right)}{\sinh \left(\lambda_{a}-\mu_{b}+i \gamma\right)}\right) .
$$

The special case of the formula (3.11), when both states correspond to the same Betheeigenvector, ${ }^{15}$ gives the Gaudin formula:

$$
\langle\vec{\lambda} \mid \vec{\lambda}\rangle=\frac{\prod_{j=1}^{m} \prod_{k=1}^{m} \sinh \left(\lambda_{j}-\lambda_{k}-i \gamma\right)}{\prod_{j>k} \sinh \left(\lambda_{k}-\lambda_{j}\right) \sinh \left(\lambda_{j}-\lambda_{k}\right)} \cdot \operatorname{det} \Phi(\vec{\lambda}),
$$

where $\Phi(\vec{\lambda})$ is the Gaudin-matrix, which can be obtained from the counting-function $(2.22)$ as follows:

$$
\Phi_{a b}(\vec{\lambda})=-i \frac{\partial}{\partial \lambda_{b}} Z_{\lambda}\left(\lambda_{a} \mid \vec{\lambda}\right), \quad a, b=1, . ., m,
$$

\footnotetext{
${ }^{14}$ We just note that the factors coming from the $(A+D)$-wings of $(3.7)$ give scalar factors since the sandwiching states are eigenstates of $(A+D)$.

${ }^{15}$ If the two eingenstates are different the scalar product is zero.
} 
where we indicated, that the counting-function should be considered as a function of the Bethe-roots. This Bethe-root dependence can be read off from (2.22).

From (3.12) it can be seen, that the matrix element $H_{a b}(\vec{\mu} \mid \vec{\lambda})$ depends on only one single component of the vector $\vec{\mu}$. This observation makes possible remarkable simplifications, when diagonal form-factors are computed. In this case one needs to compute scalar products, when the components of the vector $\vec{\mu}$ take values either from the set of Bethe-roots $\left\{\lambda_{j}\right\}_{j=1, . . m}$ or from the set of inhomogeneities $\left\{\xi_{k}\right\}_{k=1, . . N}$ of the model. In these cases the matrix elements of $H(\vec{\mu} \mid \vec{\lambda})$ take the form:

$$
\begin{aligned}
\left.H_{a b}(\vec{\mu} \mid \vec{\lambda})\right|_{\mu_{b} \rightarrow \lambda_{c}} & =(-1)^{m-1} \prod_{j=1}^{m} \sinh \left(\lambda_{c}-\lambda_{j}-i \gamma\right) \Phi_{a c}(\vec{\lambda}), \quad a, b, c=1, . ., m . \\
\left.\frac{1}{r\left(\mu_{b}\right)} H_{a b}(\vec{\mu} \mid \vec{\lambda})\right|_{\mu_{b} \rightarrow \xi_{c}} & =\frac{(-1)^{m} \sinh (-i \gamma) \prod_{j=1}^{m} \sinh \left(\xi_{c}-\lambda_{j}+i \gamma\right)}{\sinh \left(\lambda_{a}-\xi_{c}\right) \sinh \left(\lambda_{a}-\xi_{c}-i \gamma\right)}, \quad a, b, c=1, . ., m .
\end{aligned}
$$

\section{The computation of $\left\langle e_{n}\right\rangle_{\lambda}$}

Now we are in the position to compute $\left\langle e_{n}\right\rangle_{\lambda}$ on the lattice. First the contribution of the eigenvalues of the transfer-matrices are lifted:

$$
\left\langle e_{n}\right\rangle_{\lambda}=\prod_{k=1}^{m} \frac{\sinh \left(\xi_{n}-\lambda_{k}\right)}{\sinh \left(\xi_{n}-\lambda_{k}+i \gamma\right)} \cdot\left\langle D\left(\xi_{n}\right)\right\rangle_{\lambda} .
$$

As a consequence of (3.8) the expectation value $\left\langle D\left(\xi_{n}\right)\right\rangle_{\lambda}$ can be written as:

$$
\left\langle D\left(\xi_{n}\right)\right\rangle_{\lambda}=\frac{\left\langle\vec{\lambda}\left|D\left(\xi_{n}\right)\right| \vec{\lambda}\right\rangle}{\langle\vec{\lambda} \mid \vec{\lambda}\rangle}=\sum_{A=1}^{m} \frac{1}{r\left(\lambda_{A}\right)} \frac{\prod_{k=1}^{m} \sinh \left(\lambda_{A}-\lambda_{k}-i \gamma\right)}{\sinh \left(\lambda_{A}-\xi_{n}\right) \prod_{\substack{k=1 \\ k \neq A}}^{m} \sinh \left(\lambda_{A}-\lambda_{k}\right)} \cdot \frac{\langle\vec{\mu}(A) \mid \vec{\lambda}\rangle}{\langle\vec{\lambda} \mid \vec{\lambda}\rangle}
$$

where $\mu^{(A)}$ is an $m$-component vector, which differs from $\vec{\lambda}$ only in its $A$ th component, which is equal to the inhomogeneity corresponding to the $n$th site:

$$
\mu_{k}^{(A)}=\left\{\begin{array}{ll}
\lambda_{k}, & k \neq A, \\
\xi_{n}, & k=A .
\end{array} \quad k=1, . ., m\right.
$$

Due to $(3.15)$ and (3.16) using some simple determinant identities, $\left\langle\vec{\mu}^{(A)} \mid \vec{\lambda}\right\rangle$ can be written as:

$$
\left\langle\vec{\mu}^{(A)} \mid \vec{\lambda}\right\rangle=\prod_{j=1}^{m} \frac{\sinh \left(\xi_{n}-\lambda_{j}+i \gamma\right)}{\sinh \left(\xi_{n}-\lambda_{j}-i \gamma\right)} \cdot \frac{\prod_{b=1}^{m} \prod_{j=1}^{m} \sinh \left(\mu_{b}^{(A)}-\lambda_{j}-i \gamma\right) \cdot \operatorname{det} \hat{\mathcal{H}}\left(\vec{\mu}^{(A)} \mid \vec{\lambda}\right)}{\prod_{j>k}^{m} \sinh \left(\mu_{k}^{(A)}-\mu_{j}^{(A)}\right) \sinh \left(\lambda_{j}-\lambda_{k}\right)},
$$


where the $m \times m$ matrix $\hat{\mathcal{H}}\left(\vec{\mu}^{(A)} \mid \vec{\lambda}\right)$ is given by:

$$
\hat{\mathcal{H}}_{a b}\left(\vec{\mu}^{(A)} \mid \vec{\lambda}\right)= \begin{cases}\tilde{\Phi}_{a b}(\vec{\lambda}), & b \neq A \\ \mathcal{V}_{a} \equiv \frac{-\sinh (-i \gamma)}{\sinh \left(\lambda_{a}-\xi_{n}\right) \sinh \left(\lambda_{a}-\xi_{n}-i \gamma\right)}, & b=A\end{cases}
$$

Here

$$
\tilde{\Phi}_{a b}(\vec{\lambda})=\Phi_{a b}(\vec{\lambda}) \frac{1}{r\left(\lambda_{b}\right)}, \quad a, b=1, . ., m .
$$

As a consequence of $(3.10) \operatorname{det} \Phi(\vec{\lambda})=\operatorname{det} \tilde{\Phi}(\vec{\lambda})$, thus in $(3.13)$ the $\Phi(\vec{\lambda}) \rightarrow \tilde{\Phi}(\vec{\lambda})$ replacement can be done. Using (4.3), (4.4) and (4.5) one obtains:

$$
\frac{\left\langle\vec{\mu}^{(A)} \mid \vec{\lambda}\right\rangle}{\langle\vec{\lambda} \mid \vec{\lambda}\rangle}=\prod_{\substack{j=1 \\ j \neq A}}^{m} \frac{\sinh \left(\lambda_{A}-\lambda_{j}\right)}{\sinh \left(\xi_{n}-\lambda_{j}\right)} \cdot \prod_{j=1}^{m} \frac{\sinh \left(\xi_{n}-\lambda_{j}+i \gamma\right)}{\sinh \left(\lambda_{A}-\lambda_{j}-i \gamma\right)} r\left(\lambda_{A}\right)\left(\Phi^{-1}(\vec{\lambda}) \cdot \hat{\mathcal{H}}\left(\vec{\mu}^{(A)} \mid \vec{\lambda}\right)\right)_{A A},
$$

where apart from simplifying the multiplicative factors, we used (3.13) with the $\Phi \rightarrow \tilde{\Phi}$ replacement and computed the ratio of the determinants of $\hat{\mathcal{H}}\left(\vec{\mu}^{(A)} \mid \vec{\lambda}\right)$ and $\tilde{\Phi}(\vec{\lambda})$ as the determinant of $\tilde{\Phi}^{-1}(\vec{\lambda}) \cdot \hat{\mathcal{H}}\left(\vec{\mu}^{(A)} \mid \vec{\lambda}\right)$. As a consequence of (4.5), the latter matrix differs from the unity matrix only in its $A$ th column. Thus its determinant could also be computed as an expression of matrix elements of $\tilde{\Phi}^{-1}(\vec{\lambda})$ and $\hat{\mathcal{H}}\left(\vec{\mu}^{(A)} \mid \vec{\lambda}\right)$.

Inserting (4.7) into (4.2) and the result into (4.1) one ends up with the simple result:

$$
\left\langle e_{n}\right\rangle_{\lambda}=-\sum_{A=1}^{m}\left(\Phi^{-1}(\vec{\lambda}) \cdot \hat{\mathcal{H}}\left(\vec{\mu}^{(A)} \mid \vec{\lambda}\right)\right)_{A A} .
$$

Using (4.5), this can be written in components as:

$$
\left\langle e_{n}\right\rangle_{\lambda}=-\sum_{a=1}^{m} \sum_{b=1}^{m} \Phi_{a b}^{-1}(\vec{\lambda}) \mathcal{V}_{b}=-\sum_{a=1}^{m} S_{a}
$$

where $S_{a}$ is the solution of the set of linear equations:

$$
\sum_{b=1}^{m} \Phi_{a b}(\vec{\lambda}) S_{b}=\mathcal{V}_{a}, \quad a=1, . ., m
$$

\subsection{The determination of $S_{a}$}

In this subsection we show that equation (4.10) for the vector $S_{a}$ can be formulated as a set of linear-integral-equations containing the counting-function of the model. The advantage of this formulation is that it allows one to take the continuum limit in a straightforward manner.

The first step is to compute the matrix elements of $\Phi(\vec{\lambda})$ from $(3.14)$ :

$$
\Phi_{a b}(\vec{\lambda})=-i Z_{\lambda}^{\prime}\left(\lambda_{a}\right) \delta_{a b}-2 \pi i K\left(\lambda_{a}-\lambda_{b} \mid \gamma\right), \quad a, b=1, . . m
$$

where

$$
K(\lambda \mid \gamma)=\frac{1}{2 \pi} \frac{\sin (2 \gamma)}{\sinh (\lambda-i \gamma) \sinh (\lambda+i \gamma)}
$$


Now an important remark is in order. From (4.11) it can be seen, that apart form the $\delta_{a b}$ term, the $a b$ matrix element of $\Phi(\vec{\lambda})$ is given by a function of two variables taken at the arguments $\lambda_{a}$ and $\lambda_{b}$, and similarly from (4.5) it is obvious that $\mathcal{V}_{a}$ is an analytic function taken at the position $\lambda_{a}$. This suggests that the components of the unknown vector $S_{a}$ should be sought in the following form:

$$
S_{a}=X\left(\lambda_{a}\right), \quad a=1, \ldots, m,
$$

where $X(\lambda)$ is supposed to be an analytic (meromorphic) function on the complex-plane. ${ }^{16}$ The advantage of such an Ansatz becomes obvious in the large $N$-limit, because summation for the large number of components becomes a convolution integral plus a remnant sum. ${ }^{17}$

To transform the sum in (4.10) into an integral we should use the following lemma $[6,8]$.

Lemma. Let $\left\{\lambda_{j}\right\}_{j=1, . ., m}$ solutions of the Bethe-equations (2.17) and let $f(\lambda)$ a meromorphic function, which is continuous and bounded on the real axis. Denote $p^{(f)}$ its pole located the closest to the real axis. Then for $|\operatorname{Im} \mu|<\left|\operatorname{Im} p^{(f)}\right|$ the following equation holds:

$$
\begin{aligned}
\sum_{j=1}^{m} f\left(\mu-\lambda_{j}\right)= & \sum_{j=1}^{m_{C}} f\left(\mu-c_{j}\right)-\sum_{j=1}^{m_{H}} f\left(\mu-h_{j}\right)+\int_{-\infty}^{\infty} \frac{d \lambda}{2 \pi} f(\mu-\lambda) Z_{\lambda}^{\prime}(\lambda)- \\
& -\sum_{\alpha= \pm_{-\infty}} \int_{-\infty}^{\infty} \frac{d \lambda}{2 \pi} f(\mu-\lambda-i \alpha \eta) Z_{\lambda}^{\prime}(\lambda+i \alpha \eta) \mathcal{F}_{\alpha}^{(\lambda)}(\lambda+i \alpha \eta),
\end{aligned}
$$

where

$$
\mathcal{F}_{ \pm}^{(\lambda)}(\lambda)=\frac{(-1)^{\delta} e^{ \pm i Z_{\lambda}(\lambda)}}{1+(-1)^{\delta} e^{ \pm i Z_{\lambda}(\lambda)}}
$$

furthermore $h_{j}$ and $c_{j}$ denote the positions of holes and complex Bethe-roots respectively. $\eta$ is a small positive contour-integral parameter which should satisfy the inequalities:

$$
0<\eta<\min \left\{\left|\operatorname{Im} p_{\lambda}^{ \pm}\right|\right\}, \quad|\operatorname{Im} \mu \pm \eta|<\left|\operatorname{Im} p^{(f)}\right|,
$$

where $p_{\lambda}^{ \pm}$denotes those complex ${ }^{18}$ poles of $\mathcal{F}_{ \pm}^{(\lambda)}(\lambda)$, which are located the closest to the real axis.

The summation formula (4.14) can be extended to the $|\operatorname{Im} \mu|>\left|\operatorname{Im} p^{(f)}\right|$ domain by an analytical continuation procedure being similar to the analytical continuation of the DDV equation to the whole complex plane [8].

Using the Ansatz (4.13) and the formula (4.11) together with the parameterizations (2.11), (2.12), the linear equations (4.10) take the form:

$$
-i Z^{\prime}\left(\lambda_{a}\right) X\left(\lambda_{a}\right)-2 \pi i \sum_{b=1}^{m} K\left(\lambda_{a}-\lambda_{b} \mid \gamma\right) X\left(\lambda_{b}\right)=2 \pi i K\left(\lambda_{a}-\rho_{n} \mid \frac{\gamma}{2}\right), \quad a=1, . ., m,
$$

\footnotetext{
${ }^{16}$ The thermodynamic limit for the ground state expectation value was treated by the same tacit assumption in [18].

${ }^{17}$ The convolution integral comes from the "Dirac-see" of real roots and the remnant sum is related to finite number of excitations above this see.

${ }^{18}$ I.e. not real.
} 
where $\rho_{n}=+\rho$ if $n$ is even and $\rho_{n}=-\rho$ otherwise with $\rho$ given by (2.13). We transform (4.17) into integral equations with the help of (4.14). Since in (4.14) the integrand always contains a factor $Z_{\lambda}^{\prime}(\lambda)$, it is convenient to parameterize the function $X(\lambda)$ as:

$$
X(\lambda)=\frac{\mathcal{G}(\lambda)}{Z_{\lambda}^{\prime}(\lambda)}
$$

Then using (4.14), for the case of pure hole states, the linear equations can be rewritten in the form of the following linear set of integral equations:

$$
\begin{aligned}
& \mathcal{G}(\lambda)+\int_{-\infty}^{\infty} d \lambda^{\prime} K\left(\lambda-\lambda^{\prime} \mid \gamma\right) \mathcal{G}\left(\lambda^{\prime}\right)- \\
& -\sum_{\alpha= \pm} \int_{-\infty}^{\infty} d \lambda^{\prime} K\left(\lambda-\lambda^{\prime}-i \alpha \eta \mid \gamma\right) \mathcal{G}\left(\lambda^{\prime}+i \alpha \eta\right) \mathcal{F}_{\alpha}^{(\lambda)}\left(\lambda^{\prime}+i \alpha \eta\right)= \\
& \quad=-2 \pi K\left(\lambda-\rho_{n} \mid \frac{\gamma}{2}\right)+\sum_{j=1}^{m_{H}} K\left(\lambda-h_{j} \mid \gamma\right) X_{j},
\end{aligned}
$$

where

$$
X_{j}=X\left(h_{j}\right), \quad j=1, \ldots, m_{H},
$$

such that they should satisfy the discrete set of equations:

$$
X_{j}=\frac{\mathcal{G}\left(h_{j}\right)}{Z_{\lambda}^{\prime}\left(h_{j}\right)}, \quad j=1, \ldots, m_{H} .
$$

Similarly, (4.14) allows us to rephrase (4.9) as:

$$
\left\langle e_{n}\right\rangle_{\lambda}=\sum_{j=1}^{m_{H}} X_{j}-\int_{-\infty}^{\infty} \frac{d \lambda}{2 \pi} \mathcal{G}(\lambda)+\sum_{\alpha= \pm} \int_{-\infty}^{\infty} \frac{d \lambda}{2 \pi} \mathcal{G}(\lambda+i \alpha \eta) \mathcal{F}_{\alpha}^{(\lambda)}(\lambda+i \alpha \eta) .
$$

Acting $^{19}(1+K)^{-1}$ on (4.19), the equations take the form:

$$
\begin{aligned}
\mathcal{G}(\lambda) & -\sum_{\alpha= \pm} \int_{-\infty}^{\infty} d \lambda^{\prime} G_{\lambda}\left(\lambda-\lambda^{\prime}-i \alpha \eta\right) \mathcal{G}\left(\lambda^{\prime}+i \alpha \eta\right) \mathcal{F}_{\alpha}^{(\lambda)}\left(\lambda^{\prime}+i \alpha \eta\right)= \\
& =-\frac{\pi}{\gamma} \frac{1}{\cosh \left(\frac{\pi}{\gamma}\left(\lambda-\rho_{n}\right)\right)}+\sum_{j=1}^{m_{H}} 2 \pi G_{\lambda}\left(\lambda-h_{j}\right) X_{j}
\end{aligned}
$$

where $G_{\lambda}(\lambda)$ is related to the kernel of DDV equation (2.26) by:

$$
G_{\lambda}(\lambda)=\frac{1}{2 \gamma} G\left(\frac{\pi}{\gamma} \lambda\right), \quad \text { with } \quad \gamma=\frac{\pi}{p+1} .
$$

\footnotetext{
${ }^{19}$ Appendix A. contains some Fourier-transforms, which are necessary to do these computations.
} 
With the help of the integrated form of (4.23), the term $\int_{-\infty}^{\infty} \frac{d \lambda}{2 \pi} \mathcal{G}(\lambda)$ can be eliminated from (4.22). Finally, one ends up with the following formula for the expectation value:

$$
\begin{aligned}
\left\langle e_{n}\right\rangle_{\lambda} & =\frac{1}{2}-\frac{1}{2}\left\langle\sigma_{n}^{z}\right\rangle_{\lambda} \\
\frac{1}{2}\left\langle\sigma_{n}^{z}\right\rangle_{\lambda} & =-\frac{1}{2\left(1-\frac{\gamma}{\pi}\right)}\left\{\sum_{j=1}^{m_{H}} X_{j}+\sum_{\alpha= \pm} \int_{-\infty}^{\infty} \frac{d \lambda}{2 \pi} \mathcal{G}(\lambda+i \alpha \eta) \mathcal{F}_{\alpha}^{(\lambda)}(\lambda+i \alpha \eta)\right\} .
\end{aligned}
$$

Equations (4.23), (4.21) and (4.25) constitute our final lattice results, which serve as the starting point to compute the continuum limit of the expectation values of the $\mathrm{U}(1)$ current of the MT/SG theories.

\section{The continuum limit}

The continuum limit is the appropriate $N \rightarrow \infty$ of our equations. Using (2.13), the equations (4.21), (4.23) and (4.25) can be expanded at large $N$ in a series of $\frac{1}{N}$, such that the leading power is $\frac{1}{N}$. From (2.40) and (2.43) one can see, that the continuum result will be proportional to this leading order coefficient:

$$
\mathcal{G}^{\text {cont }}(\lambda) \sim \lim _{N \rightarrow \infty} N \mathcal{G}(\lambda), \quad X_{j}^{\text {cont }} \sim \lim _{N \rightarrow \infty} N X_{j}
$$

All equations we have for the computation of the expectation value of $\sigma_{n}^{z}$ are linear. This is why using (2.40) and (2.43), one can take their appropriate linear combinations to get the continuum expressions corresponding to the components of the $\mathrm{U}(1)$ current. The equations in the continuum limit and in rapidity convention take the form:

$$
\begin{gathered}
\mathcal{G}^{(\mu)}(\theta)-\sum_{\alpha= \pm} \int_{-\infty}^{\infty} \frac{d \theta^{\prime}}{2 \pi} G\left(\theta-\theta^{\prime}-i \alpha \eta\right) \mathcal{G}^{(\mu)}\left(\theta^{\prime}+i \alpha \eta\right) \mathcal{F}_{\alpha}\left(\theta^{\prime}+i \alpha \eta\right)= \\
=-\mathcal{K}_{\mu}(\theta)+\sum_{j=1}^{m_{H}} G\left(\theta-H_{j}\right) X_{j}^{(\mu)} \\
X_{j}^{(\mu)}=\frac{\mathcal{G}^{(\mu)}\left(H_{j}\right)}{Z^{\prime}\left(H_{j}\right)}, \quad j=1, \ldots, m_{H}, \quad \mu=0,1 . \\
\left\langle j_{\mu}(x)\right\rangle_{H}=-\frac{p+1}{p}\left\{\sum_{j=1}^{m_{H}} X_{j}^{(\mu)}+\sum_{\alpha= \pm} \int_{-\infty}^{\infty} \frac{d \theta^{\prime}}{2 \pi} \mathcal{G}^{(\mu)}\left(\theta^{\prime}+i \alpha \eta\right) \mathcal{F}_{\alpha}\left(\theta^{\prime}+i \alpha \eta\right)\right\}
\end{gathered}
$$

where

$$
\mathcal{F}_{ \pm}(\theta)=\frac{(-1)^{\delta} e^{ \pm i Z(\theta)}}{1+(-1)^{\delta} e^{ \pm i Z(\theta)}}
$$

and the operator dependent source term reads as:

$$
\mathcal{K}_{\mu}(\theta)= \begin{cases}\mathcal{M} \cosh (\theta), & \mu=0 \\ \mathcal{M} \sinh (\theta), & \mu=1\end{cases}
$$


Here the index $\mu=0,1$ corresponds to the lower index of the current $J_{\mu}, G(\theta)$ is the kernel (2.26) of the DDV equation, and $\eta$ is a small positive contour integral parameter which must satisfy the inequalities:

$$
0<\eta<\min \left\{\left|\operatorname{Im} p_{j}^{( \pm)}\right|\right\}, \quad|\operatorname{Im} \theta \pm \eta|<\min (1, p) \pi,
$$

where $p_{j}^{( \pm)}$denotes those poles of $\mathcal{F}_{ \pm}(\theta)$ which are not real.

At the notation of the expectation value, we denoted that in the continuum limit we think of the state as if it was characterized by the holes. For completeness we give how the continuum quantities of (5.2) are related to those of the lattice:

$$
\begin{aligned}
& \mathcal{G}^{(0)}(\theta)=\lim _{N \rightarrow \infty} \frac{N}{2 L}\left\{\frac{\gamma}{\pi} \mathcal{G}^{\left(e_{2 n}\right)}\left(\frac{\gamma}{\pi} \theta\right)+\frac{\gamma}{\pi} \mathcal{G}^{\left(e_{2 n-1}\right)}\left(\frac{\gamma}{\pi} \theta\right)\right\}, \\
& \mathcal{G}^{(1)}(\theta)=\lim _{N \rightarrow \infty} \frac{N}{2 L}\left\{\frac{\gamma}{\pi} \mathcal{G}^{\left(e_{2 n}\right)}\left(\frac{\gamma}{\pi} \theta\right)-\frac{\gamma}{\pi} \mathcal{G}^{\left(e_{2 n-1}\right)}\left(\frac{\gamma}{\pi} \theta\right)\right\},
\end{aligned}
$$

where at the right hand side we indicated as an upper index the lattice operator, the unknown of whose linear problem ${ }^{20}$ should be considered. Finally we note that formula (5.3) for $\left\langle j_{\mu}(x)\right\rangle_{H}$ is not the final answer to the expectation value of the $\mathrm{U}(1)$ current. In the next subsection at the investigation of the charge density, it will turn out that $\left\langle j_{\mu}(x)\right\rangle_{H}$ is still not the real expectation value of the $\mathrm{U}(1)$ current in the quantum field theory, but it should be modified with an appropriate renormalization factor.

\subsection{The solution of the equations}

In this section we relate the equations (5.2) describing the expectation values of the $\mathrm{U}(1)$ current to the counting-function of the DDV-equation (2.31) corresponding to the sandwiching state. Indeed it turns out that the solutions of (5.2) are related to certain derivatives of $Z(\theta)$. The solutions we get, imply the relation (2.44).

\subsubsection{The charge density case}

Let us start with the $\mu=0$ case, which corresponds to the expectation value of the charge density. Comparing (5.2) with the derivative of the DDV equation (2.31) with respect to $\theta$, it turns out that the solution of (5.2) can be expressed in terms of the counting-function by the formulas as follows:

$$
\begin{aligned}
\mathcal{G}^{(0)}(\theta) & =-\frac{1}{L} Z^{\prime}(\theta), \\
X_{j}^{(0)} & =-\frac{1}{L}, \quad j=1, . ., m_{H} .
\end{aligned}
$$

Inserting (5.8) into (5.3), one obtains the following result for the expectation value of $j_{0}$ between $m_{H}$ solitons:

$$
\left\langle j_{0}(x)\right\rangle_{H}=\frac{p+1}{p} \frac{m_{H}}{L} .
$$

This formula requires some explanation. In the quantum field theory each soliton carries topological charge $Q=+1$. The expectation value of the topological charge in an $m_{H}$

\footnotetext{
${ }^{20}$ Here by linear problem we mean, the linear problem which enables one to compute the expectation value. Namely, the set of equations: (4.21) and (4.23), (4.25).
} 
soliton state is $\langle Q\rangle_{H}=m_{H}$. Since the charge is the integral of the charge density operator, whose expectation value has no space-time dependence, in the continuum theory the expectation value of the charge density should be $\frac{m_{H}}{L}$. It can be seen, that the result in (5.9) agrees with the expected one apart from a global coupling dependent factor of $\frac{p+1}{p}$. We got (5.9) by identifying the topological charge of the continuum field theory with twice the z-component of the spin of the lattice model, $Q \sim 2 S_{z}$. In view of the quantum field theory interpretation, formula (5.9) suggests that instead of (2.40) and (2.43) the correct identification between the lattice and continuum operators is given by the formulas:

$$
\begin{aligned}
& \left.J_{0}(x)\right|_{x=n a}=\left.\frac{p}{p+1} j_{0}(x)\right|_{x=n a}=\frac{p}{p+1} \frac{N}{L} \frac{\sigma_{2 n}^{z}+\sigma_{2 n-1}^{z}}{2}, \\
& \left.J_{1}(x)\right|_{x=n a}=\left.\frac{p}{p+1} j_{1}(x)\right|_{x=n a}=\frac{p}{p+1} \frac{N}{L} \frac{\sigma_{2 n}^{z}-\sigma_{2 n-1}^{z}}{2} .
\end{aligned}
$$

Consequently, we conclude that $\left\langle j_{\mu}(x)\right\rangle_{H}$ given in (5.2) is not the final answer in the quantum field theory (QFT), because it has to be modified by the renormalization factor $Z_{p}=\frac{p}{p+1}$. Thus, the real QFT result is given by:

$$
\left\langle J_{\mu}(x)\right\rangle_{H}=-\sum_{j=1}^{m_{H}} X_{j}^{(\mu)}-\sum_{\alpha= \pm} \int_{-\infty}^{\infty} \frac{d \theta^{\prime}}{2 \pi} \mathcal{G}^{(\mu)}\left(\theta^{\prime}+i \alpha \eta\right) \mathcal{F}_{\alpha}\left(\theta^{\prime}+i \alpha \eta\right)
$$

To summarize: (5.2) and (5.11) constitutes our final equations for computing the diagonal matrix elements of $J_{\mu}(x)$.

\subsubsection{The case of $J_{1}(x)$}

The equations (5.2) also for $\mu=1$ are related to a certain derivative of the countingfunction. The counting-function depends on the spectral parameter $\theta$, on $\ell=\mathcal{M} L$ the dimensionless length of the system and on the hole positions, ${ }^{21}$ which are also $\ell$ dependent. Then, differentiating (2.31) with respect to $\ell$, one can recognize that $\mathcal{G}^{(1)}(\theta)$ of $(5.2)$ is related to the $\ell$-derivative of the counting-function as follows:

$$
\begin{aligned}
\mathcal{G}^{(1)}(\theta) & =-\mathcal{M} \frac{d}{d \ell} Z(\theta \mid \vec{H}(\ell), \ell), \\
X_{j}^{(1)} & =\mathcal{M} H_{j}^{\prime}(\ell), \quad j=1, . ., m_{H},
\end{aligned}
$$

where we explicitly wrote out the $H_{j}$ and $\ell$ dependence of $Z(\theta)$. With the help of (5.12), one can show that $\left\langle J_{1}(x)\right\rangle_{H}$ can also be rephrased as the $\ell$-derivative of a quantity, which can be expressed directly in terms of the solution of the DDV equations;

$$
\begin{aligned}
\left\langle J_{1}(x)\right\rangle_{H} & =-\mathcal{M} \frac{d}{d \ell} \Lambda_{1}(\ell) \\
\Lambda_{1}(\ell) & =\sum_{j=1}^{m_{H}} H_{j}(\ell)-\int_{-\infty}^{\infty} \frac{d \theta}{2 \pi i}\left\{L_{+}(\theta+i \eta)-L_{-}(\theta-i \eta)\right\} .
\end{aligned}
$$

\footnotetext{
${ }^{21}$ Specifying the state, the quantum numbers of holes in the continuum version of (2.28) are fixed. I.e. They are $\ell$ independent.
} 
We note, that in (5.13) $L_{+}$and $L_{-}$have different signs under the integration. This fact has a remarkable consequence concerning the TBA description of this expectation value. Namely, if one considers the TBA description $[45,46]$ of the model at the points $1<p \in \mathbb{Z}_{+}$, where the system is described by a $\mathcal{D}_{p+1}$-type TBA-system, then it becomes obvious, that (5.13) cannot be expressed in terms of the Y-functions corresponding to the massive TBA node. ${ }^{22}$ This implies, that the TBA conjectures [38-42] for purely elastic scattering theories, cannot be valid in this non-diagonally scattering theory. Earlier a similar conclusion has been drawn in [53].

\section{The large volume expansion}

In this section we solve our equations (5.2) in the context of a systematic large volume expansion. The actual form of the representation we get, is very similar to those conjectured for purely elastic scattering theories [38-42]. Nevertheless, since our model is not a diagonally scattering theory, our large volume series differs from these TBA conjectures.

In this section we will strongly rely on the method described in [42] for the computation of the diagonal matrix elements of the trace of the stress-energy tensor in purely elastic scattering theories. We can do this, because the DDV equation (2.31) is formally similar to the TBA-equations of a purely elastic scattering theory containing two types of particles. To clarify this analogy better, as a first step we reformulate the DDV equation as a twocomponent TBA equation. Eq. (2.31) contains $Z(\theta)$ along three different lines; along the real line and on the lines $\theta \pm i \eta$ with $\theta \in \mathbb{R}$. When solving the equations, one has to compute $Z(\theta)$ on all these 3 lines. To get a closed set of equations, we have to consider (2.31) with left-hand sides $Z(\theta \pm i \eta)$ as well. The two equations for $Z(\theta \pm i \eta)$ formally look like a two component TBA equation of a diagonally scattering theory. Let $\varepsilon_{ \pm}(\theta)=Z(\theta \pm i \eta)$ and $\mathcal{L}_{ \pm}(\theta)=\ln \left(1+(-1)^{\delta} e^{ \pm i \varepsilon_{ \pm}(\theta)}\right)$, then the TBA-like form of (2.31) reads as:

$$
\varepsilon_{\alpha}(\theta)=\mathcal{S}_{\alpha}(\theta)+\sum_{\beta= \pm-\infty} \int_{-\infty}^{\infty} \frac{d \theta^{\prime}}{2 \pi} \varphi_{\alpha \beta}\left(\theta-\theta^{\prime}\right) \mathcal{L}_{\beta}\left(\theta^{\prime}\right), \quad \alpha= \pm,
$$

where $\mathcal{S}_{\alpha}(\theta)$ is the source term:

$$
\mathcal{S}_{\alpha}(\theta)=\ell \sinh (\theta+i \alpha \eta)+\sum_{k=1}^{m_{H}} \chi\left(\theta+i \alpha \eta-H_{k}\right),
$$

and

$$
\varphi_{\alpha \beta}(\theta)=i G(\theta+i(\alpha-\beta) \eta), \quad \alpha, \beta= \pm,
$$

is a symmetric matrix kernel. From the point of view of our later computations, the fact that the different quantities in (6.1) are complex, does not matter. The only important property is that the kernel (6.3) is symmetric, i.e. $\varphi_{\alpha \beta}(\theta)=\varphi_{\beta \alpha}(-\theta)$.

\footnotetext{
${ }^{22}$ This is so, because the relation between $L_{ \pm}$and $Y_{1}$ the massive Y-function is given by [46]: $L_{+}(\theta+$ $\left.i \frac{\pi}{2}\right)+L_{-}\left(\theta-i \frac{\pi}{2}\right)=\ln \left(1+Y_{1}(\theta)\right)$.
} 
In a completely analogous way the linear equations (5.2) can also be rephrased by considering them along the lines $\theta \pm i \eta$. In (5.2) the left-hand side describes the action of the linear operator on the unknown function and the right-hand side is the source term of the linear problem. Since these equations are linear, it is worth to consider the solutions of (5.2) with different "elementary" source terms, from which the solution of the physical problem can be obtained by linear combinations. Consider in general the linear problems:

$$
\mathcal{G}_{A}^{[\alpha]}(\theta)-\sum_{\beta= \pm} \int_{-\infty}^{\infty} \frac{d \theta^{\prime}}{2 \pi} \psi_{\alpha \beta}\left(\theta-\theta^{\prime}\right) \mathcal{G}_{A}^{[\beta]}\left(\theta^{\prime}\right) \mathcal{F}_{\beta}^{[\beta]}\left(\theta^{\prime}\right)=f_{A}^{[\alpha]}(\theta), \quad \alpha= \pm,
$$

where for any function $f(\theta)$ we introduced the notation: $f^{[ \pm]}(\theta)=f(\theta \pm i \eta)$, and $\psi_{\alpha \beta}(\theta)=\frac{1}{i} \varphi_{\alpha \beta}(\theta)$ is a symmetric kernel. In (6.4) $f_{A}^{[\alpha]}(\theta)$ denotes the elementary source term indexed by $A$.

If the argument of the "elementary" solution is not shifted, we denote it simply $\mathcal{G}_{A}(\theta)$ and it satisfies the equations:

$$
\mathcal{G}_{A}(\theta)-\sum_{\beta= \pm} \int_{-\infty}^{\infty} \frac{d \theta^{\prime}}{2 \pi} \psi_{\alpha \beta}\left(\theta-\theta^{\prime}-i \alpha \eta\right) \mathcal{G}_{A}^{[\beta]}\left(\theta^{\prime}\right) \mathcal{F}_{\beta}^{[\beta]}\left(\theta^{\prime}\right)=f_{A}(\theta), \quad \alpha= \pm .
$$

The elementary solutions from which the physical solutions of (5.2) and (5.11) can be combined are characterized by their source terms in (6.5) and they are as follows:

$$
\begin{array}{rlrl}
\mathcal{G}_{\mathcal{K}_{\mu}}(\theta) & \leftrightarrow f_{\mathcal{K}_{\mu}}(\theta)=\mathcal{K}_{\mu}(\theta), & & \mu=0,1 . \\
\mathcal{G}_{j}(\theta) & \leftrightarrow \quad f_{j}(\theta)=-G\left(\theta-H_{j}\right), & & j=1, \ldots, m_{H}, \\
\mathcal{G}_{u}(\theta) & \leftrightarrow f_{u}(\theta)=1 . &
\end{array}
$$

As a consequence of equations (6.4), for any pair of indexes the following identities hold:

$$
\sum_{\alpha= \pm} \int_{-\infty}^{\infty} \frac{d \theta}{2 \pi} f_{A}^{[\alpha]}(\theta) \mathcal{G}_{B}^{[\alpha]}(\theta) \mathcal{F}_{\alpha}^{[\alpha]}(\theta)=\sum_{\alpha= \pm} \int_{-\infty}^{\infty} \frac{d \theta}{2 \pi} f_{B}^{[\alpha]}(\theta) \mathcal{G}_{A}^{[\alpha]}(\theta) \mathcal{F}_{\alpha}^{[\alpha]}(\theta) .
$$

Now we show how the exact Gaudin-matrix enters the large volume expansion and how one can express the solutions of (5.2) in terms of the elementary solutions (6.6)-(6.8). First, we consider the integral equation in $(5.2)$ as if $X_{j}^{(\mu)}$ s were arbitrary parameters. Then using (6.4) and (6.6)-(6.8) the solution can be written as:

$$
\mathcal{G}^{(\mu)}(\theta)=-\mathcal{G}_{\mathcal{K}_{\mu}}(\theta)-\sum_{j=1}^{m_{H}} \mathcal{G}_{j}(\theta) X_{j}^{(\mu)} .
$$

However we know from (5.2) that $X_{j}^{(\mu)}$ s are not independent from $\mathcal{G}^{(\mu)}(\theta)$, but they are related by: $\mathcal{G}^{(\mu)}\left(H_{j}\right)=X_{j}^{(\mu)} Z^{\prime}\left(H_{j}\right)$. Inserting this relation into (6.10) taken at $\theta=H_{k}$, one ends up with the discrete set of equations for $X_{j}^{(\mu)}$ as follows:

$$
\sum_{j=1}^{m_{H}}\left\{Z^{\prime}\left(H_{k}\right) \delta_{j k}+\mathcal{G}_{j}\left(H_{k}\right)\right\} X_{j}^{(\mu)}=-\mathcal{G}_{\mathcal{K}_{\mu}}\left(H_{k}\right), \quad k=1, \ldots, m_{H} .
$$


From (2.31) and (2.28), it follows that the matrix entering (6.11) is nothing, but the Gaudinmatrix of physical excitations over the Dirac-see, which we call exact Gaudin-matrix:

$$
\hat{\Phi}_{k j}(\vec{H})=\frac{d}{d H_{j}} Z\left(H_{k} \mid \vec{H}\right)=Z^{\prime}\left(H_{k}\right) \delta_{j k}+\mathcal{G}_{j}\left(H_{k}\right), \quad j, k=1, . ., m_{H} .
$$

Using (6.10), (6.11) and (6.12) finally we get:

$$
\begin{aligned}
X_{k}^{(\mu)} & =-\sum_{j=1}^{m_{H}} \hat{\Phi}_{k j}^{-1}(\vec{H}) \mathcal{G}_{\mathcal{K}_{\mu}}\left(H_{j}\right), \quad k=1, . ., m_{H} . \\
\mathcal{G}^{(\mu)}(\theta) & =-\mathcal{G}_{\mathcal{K}_{\mu}}(\theta)+\sum_{k=1}^{m_{H}} \sum_{j=1}^{m_{H}} \mathcal{G}_{k}(\theta) \hat{\Phi}_{k j}^{-1}(\vec{H}) \mathcal{G}_{\mathcal{K}_{\mu}}\left(H_{j}\right) .
\end{aligned}
$$

The last missing piece is the expression of $\left\langle J_{\mu}(x)\right\rangle_{H}$ in terms of the "elementary" solutions. This can be computed by inserting (6.13) and (6.14) into (5.11) and by using the identity:

$$
\sum_{\alpha= \pm} \int_{-\infty}^{\infty} \frac{d \theta}{2 \pi} \mathcal{G}_{j}^{[\alpha]}(\theta) \mathcal{F}_{\alpha}^{[\alpha]}(\theta)=1-\mathcal{G}_{u}\left(H_{j}\right), \quad j=1, . ., m_{H}
$$

which can be derived by using (6.9). The final result is as follows:

$$
\left\langle J_{\mu}(x)\right\rangle_{H}=\sum_{\alpha= \pm} \int_{-\infty}^{\infty} \frac{d \theta}{2 \pi} \mathcal{G}_{\mathcal{K}_{\mu}}^{[\alpha]}(\theta) \mathcal{F}_{\alpha}^{[\alpha]}(\theta)+\sum_{k=1}^{m_{H}} \sum_{j=1}^{m_{H}} \mathcal{G}_{u}\left(H_{j}\right) \hat{\Phi}_{j k}^{-1}(\vec{H}) \mathcal{G}_{\mathcal{K}_{\mu}}\left(H_{k}\right)
$$

The first term in (6.16) corresponds to the so-called vacuum contribution [41, 42]. Constructing the all order large volume solution of (6.4) for $A=\mathcal{K}_{\mu}$, it can be written as an infinite series similar to that of LeClair and Mussardo [38-40]. Performing carefully the calculations one obtains for the vacuum piece the result as follows:

$$
\begin{aligned}
\left.\left\langle J_{\mu}(x)\right\rangle_{H}\right|_{\text {vac }}=\sum_{n_{+}=0}^{\infty} \sum_{n_{-}=0}^{\infty} & \frac{1}{n_{+} ! n_{-} !} \int \prod_{i=1}^{n_{+}+n_{-}} \frac{d \theta_{i}}{2 \pi} \prod_{i=1}^{n_{+}} \mathcal{F}_{+}(\theta+i \eta) \prod_{i=n_{+}+1}^{n_{+}+n_{-}} \mathcal{F}_{-}(\theta-i \eta) \\
& \times F_{c}^{J_{\mu}}\left(\theta_{1}+i \eta, \ldots, \theta_{n_{+}}+i \eta, \theta_{n_{+}+1}-i \eta, \ldots, \theta_{n_{+}+n_{-}}-i \eta\right)
\end{aligned}
$$

where $F_{c}^{J_{\mu}}$ denotes the connected diagonal form factors of the operator $J_{\mu}(x)$ between pure soliton states. Since $J_{\mu}$ is a conserved current, its connected form-factors can be determined by simple modifications of the arguments of references [38] and [39]. The explicit form of $F_{c}^{J_{\mu}}$ is given by the compact formula: ${ }^{23}$

$$
F_{c}^{J_{\mu}}\left(\theta_{1}, \theta_{2}, \ldots, \theta_{n}\right)=\sum_{\sigma \in S_{n}} \mathcal{K}_{\mu}\left(\theta_{\sigma(n)}\right) \prod_{j=1}^{n-1} G\left(\theta_{\sigma(j)}-\theta_{\sigma(j+1)}\right)
$$

where $\sigma$ denotes the elements of the the symmetric group $S_{n}$.

\footnotetext{
${ }^{23} \operatorname{In}(6.18)$ the $\left\langle\theta \mid \theta^{\prime}\right\rangle=2 \pi \delta\left(\theta-\theta^{\prime}\right)$ normalization for the continuum states is assumed.
} 
Before turning to the second term is the r.h.s. of (6.16) it is worth to recall the conjecture of $[41,42]$ for the diagonal matrix elements of local operators in purely elastic scattering theories.

The conjecture for purely elastic scattering theories states, that the exact finite volume expectation value of any local operator $\mathcal{O}(x)$ can be written as:

$$
\begin{aligned}
\left\langle H_{1}, \ldots, H_{n}|\mathcal{O}(x)| H_{1}, \ldots, H_{n}\right\rangle= & \frac{1}{\rho\left(H_{1}, . ., H_{n}\right)} \\
& \times \sum_{\left\{H_{+}\right\} \cup\left\{H_{-}\right\}} \mathcal{D}^{\mathcal{O}}\left(\left\{H_{+}\right\}\right) \rho\left(\left\{H_{-}\right\} \mid\left\{H_{+}\right\}\right),
\end{aligned}
$$

where $\rho(\vec{H})$ is the determinant of the exact Gaudin-matrix:

$$
\rho\left(H_{1}, . ., H_{n}\right)=\operatorname{det} \hat{\Phi}(\vec{H}),
$$

the sum in (6.19) runs for all bipartite partitions of the rapidities of the sandwiching state: $\left\{H_{1}, . ., H_{n}\right\}=\left\{H_{+}\right\} \cup\left\{H_{-}\right\}$, such that

$$
\rho\left(\left\{H_{+}\right\} \mid\left\{H_{-}\right\}=\operatorname{det} \hat{\Phi}_{+}(\vec{H}),\right.
$$

with $\hat{\Phi}_{+}(\vec{H})$ being the submatrix of $\hat{\Phi}(\vec{H})$ corresponding to the subset $\left\{H_{+}\right\}$. The most important part in (6.19) is the form of the so-called dressed-form factor $\mathcal{D}^{\mathcal{O}}\left(\left\{H_{+}\right\}\right)$. It is expressed as an infinite sum in terms of the connected diagonal form-factors of the theory:

$$
\begin{aligned}
\mathcal{D}^{\mathcal{O}}\left(\left\{H_{1}, \ldots, H_{l}\right\}\right)= & \sum_{n_{1}, ., n_{k}}^{\infty} \frac{1}{\prod_{i} n_{i} !} \int_{-\infty}^{\infty} \prod_{j=1}^{\sum_{i} n_{i}} \frac{d \theta_{j}}{2 \pi\left[1+e^{\varepsilon_{\beta_{j}}\left(\theta_{j}\right)}\right]} \\
& \times F_{2 l, 2 n_{1}, . ., 2 n_{k}}^{\mathcal{O}}\left(H_{1}, . ., H_{l}, \theta_{1}, \ldots, \theta_{\sum_{i} n_{i}}\right),
\end{aligned}
$$

where $\varepsilon_{\beta_{j}}\left(\theta_{j}\right)$ is the pseudoenergy of the particle of type $\beta_{j}$ in the TBA equations of the model and $F_{2 l, 2 n_{1}, . ., 2 n_{k}}^{\mathcal{O}}$ is the connected diagonal form factor of the operator $\mathcal{O}$ in the theory, such that $n_{i}$ denotes the number of particles of type $\beta_{i}$ in the set $\left\{\theta_{1}, . ., \theta_{\sum_{i} n_{i}}\right\}$.

Now we can turn to compute the second term in the r.h.s. of (6.16). In this paper we consider the expectation values of $J_{\mu}$ between pure soliton states. In the SG/MT model solitons scatter diagonally among themselves. In this respect the pure soliton sector is very similar to a purely elastic scattering theory. Consequently, we expect a final result similar to the conjecture (6.19) for the soliton expectation values of $J_{\mu}$. This is why we will show that the second term in the right-hand side of (6.16) can be brought into the form of (6.19) such that our dressed form factors are defined as the coefficients of $\frac{\rho\left(\left\{H_{-}\right\} \mid\left\{H_{+}\right\}\right)}{\rho\left(H_{1}, \ldots, H_{n}\right)}$ in the sum. The expression we want to bring into the form of (6.19) reads as follows: ${ }^{24}$

$$
\left.\left\langle J_{\mu}(x)\right\rangle_{H}\right|_{e x}=\sum_{k=1}^{m_{H}} \sum_{j=1}^{m_{H}} \mathcal{G}_{u}\left(H_{j}\right) \hat{\Phi}_{j k}^{-1}(\vec{H}) \mathcal{G}_{\mathcal{K}_{\mu}}\left(H_{k}\right) .
$$

\footnotetext{
${ }^{24}$ The term corresponding to $\left\{H_{+}\right\}=\emptyset$ is given by the vacuum contribution (6.17).
} 
Our sandwiching state is composed of $m_{H}$-solitons. The key point in the computation is that the inverse Gaudin-matrix can be expanded in terms of its minors as follows [42, 44].

$$
\hat{\Phi}_{i j}^{-1}=\frac{\mathcal{C}_{i j}}{\operatorname{det} \hat{\Phi}}, \quad i, j=1, . ., m_{H},
$$

where $\mathcal{C}_{i j}$ is the co-factor matrix. It is given by:

$$
\mathcal{C}_{i j}=\left\{\begin{array}{lc}
\operatorname{det} \hat{\Phi}(\{i\}), & i=j, \\
\sum_{n=0}^{m_{H}-2} \sum_{\{\alpha\}}(-1)^{n+1} \hat{\Phi}_{i \alpha_{1}} \hat{\Phi}_{\alpha_{1} \alpha_{2}} \ldots \hat{\Phi}_{\alpha_{n} j} \operatorname{det} \hat{\Phi}\left(\left\{j, i, \alpha_{1}, \ldots, \alpha_{n}\right\}\right), & i \neq j,
\end{array}\right.
$$

where $\{\alpha\}=\left\{1,2, \ldots, m_{H}\right\} \backslash\{i, j\}$ and $\hat{\Phi}(\{\mathcal{I}\})$ denotes the matrix obtained by omitting from $\hat{\Phi}$ the rows and columns indexed by the set $\{\mathcal{I}\}$.

First, one has to construct the all order large volume solution ${ }^{25}$ of (6.4) for $A \in$ $\left\{u, \mathcal{K}_{0}, \mathcal{K}_{1}\right\}$ and to insert (6.24) with (6.25) into (6.23). Then after the careful bookkeeping of the terms being identical due to appropriate permutations of the variables, one obtains the following expression for the dressed form factors between soliton states for $J_{\mu}$ :

$$
\begin{aligned}
& \mathcal{D}^{J_{\mu}}\left(\left\{H_{1}, \ldots, H_{n}\right\}\right)=\sum_{n_{+}=0}^{\infty} \sum_{n_{-}=0}^{\infty} \frac{1}{n_{+} ! n_{-} !} \int^{n_{+}+n_{-}} \prod_{i=1}^{d} \frac{d \theta_{i}}{2 \pi} \prod_{i=1}^{n_{+}} \mathcal{F}_{+}\left(\theta_{i}+i \eta\right) \prod_{i=n_{+}+1}^{n_{+}+n_{-}} \mathcal{F}_{-}\left(\theta_{i}-i \eta\right) \\
& \quad \times F_{c}^{J_{\mu}}\left(H_{1}, H_{2}, \ldots, H_{n}, \theta_{1}+i \eta, \ldots, \theta_{n_{+}}+i \eta, \theta_{n_{+}+1}-i \eta, \ldots, \theta_{n_{+}+n_{-}}-i \eta\right) .
\end{aligned}
$$

Then the expectation value of $J_{\mu}$ between pure soliton states is given by a formula being completely analogous to (6.19):

$$
\begin{aligned}
\left\langle H_{1}, \ldots, H_{m_{H}}\left|\mathcal{J}_{\mu}(x)\right| H_{1}, \ldots, H_{m_{H}}\right\rangle= & \frac{1}{\rho\left(H_{1}, . ., H_{m_{H}}\right)} \\
& \times \sum_{\left\{H_{+}\right\} \cup\left\{H_{-}\right\}} \mathcal{D}^{\mathcal{J}_{\mu}}\left(\left\{H_{+}\right\}\right) \rho\left(\left\{H_{-}\right\} \mid\left\{H_{+}\right\}\right),
\end{aligned}
$$

with $\mathcal{D}^{\mathcal{J}_{\mu}}\left(\left\{H_{+}\right\}\right)$is given by (6.26). The result (6.26) requires some interpretation in view of previous results for purely elastic scattering theories [41, 42], which we summarized in (6.19). If one compares our results (6.27), (6.26) to the purely elastic TBA conjectures (6.19), (6.22), it is easy to recognize that the difference is present only in the actual form of the dressed form factors. Moreover at leading order in the volume, when the integral terms in (6.26) and (6.22) can be neglected, in accordance with [43] our formula agrees with the conjecture for purely elastic scattering theories [47, 48]. The reason for this might be, that pure soliton states form a purely elastic scattering subsector in the scattering theory of the SG/MT model. On the other hand, if one takes a look at the exponentially small in volume corrections, which are given by the integral terms in (6.26) and (6.22), it becomes obvious that (6.22) cannot describe ${ }^{26}$ the SG/MT model by simply substituting the massive pseudoenergy of the TBA equations $[45,46]$ of the SG-model

\footnotetext{
${ }^{25}$ In the actual computations it is convenient to write $\mathcal{G}_{A}\left(H_{j}\right) \rightarrow \mathcal{G}_{A}^{[ \pm]}\left(H_{j} \mp i \eta\right)$ and iterate the twocomponent equations (6.4).

${ }^{26}$ The same fact was recognized in [53].
} 
into (6.22). This is because at the level of exponentially small in volume corrections, the interactions between solitons and antisolitons will also contribute. Apart from the differences between (6.26) and (6.22) there is a remarkable similarity, too. Namely both formula contains the connected diagonal form factors of the operator sandwiched. Though we computed explicitely the diagonal matrix elements of only the components of the U(1) current of the SG/MT model, based on the remarkable similarity of our result with those obtained in purely elastic scattering theories [41, 42], we make the following conjecture:

Conjecture. For any local operator $\mathcal{O}(x)$ in the $S G / M T$ model the expectation value in an n-soliton state is given by (6.19), such that the dressed form factors are given by the formula:

$$
\begin{aligned}
& \mathcal{D}^{\mathcal{O}}\left(\left\{H_{1}, \ldots, H_{n}\right\}\right)=\sum_{n_{+}=0}^{\infty} \sum_{n_{-}=0}^{\infty} \frac{1}{n_{+} ! n_{-} !} \int^{n_{+}+n_{-}} \prod_{i=1}^{d \theta_{i}} \frac{n_{+}}{2 \pi} \prod_{i=1}^{n_{+}} \mathcal{F}_{+}\left(\theta_{i}+i \eta\right) \prod_{i=n_{+}+1}^{n_{+}+n_{-}} \mathcal{F}_{-}\left(\theta_{i}-i \eta\right) \\
& \quad \times F_{c}^{\mathcal{O}}\left(H_{1}, H_{2}, \ldots, H_{n}, \theta_{1}+i \eta, \ldots, \theta_{n_{+}}+i \eta, \theta_{n_{+}+1}-i \eta, \ldots, \theta_{n_{+}+n_{-}}-i \eta\right)
\end{aligned}
$$

where $F_{c}^{\mathcal{O}}$ denotes the connected diagonal form factors of $O(x)$ in pure soliton states and $\mathcal{F}_{ \pm}(\theta)$ are an appropriate nonlinear expressions (5.4) of the counting function of the continuum theory.

The further analytical and numerical tests of our conjecture are left for future investigations.

\section{$7 \quad$ Summary and outlook}

In this paper we argued that, through the light-cone lattice approach, the QISM admits an appropriate framework for computing the finite volume form factors of the MassiveThirring and sine-Gordon theories. We demonstrated that the QISM works efficiently, when the diagonal matrix elements of local operators are computed.

Our approach is similar to that of $[49,50]$, where the finite temperature one-point functions of all local operators of the sine-Gordon model have been computed, which corresponds to finite volume vacuum expectation values in our language. The main difference between the two approaches is that, the authors of $[49,50]$ work in a picture, when the compactified direction is time and the compactification length corresponds to the inverse temperature, while we work in the other possible channel, when the space is compactified. This allows us to consider form factors of operators between all possible excited states of the model. Consequently, our method allows one to extend the results of $[49,50]$ from vacuum expectation values to compute diagonal matrix elements of local operators of the Massive-Thirring/sine-Gordon models. To be more precise our approach works for operators, which are composed of Fermi fields and their derivatives in the MT model and for their bosonized counterparts in the SG model.

Nevertheless, in this paper we considered only a simple operator, the U(1) current of the theory and computed its diagonal matrix elements between pure soliton states. Our results are given by the formulas (5.2) and (5.11). The computation of an expectation value consists of three steps: 
1. First one should solve the DDV equation (2.31) for the sandwiching state.

2. Then, one should solve the linear equations (5.2).

3. Finally, the solution of (5.2) should be inserted into (5.11).

The whole procedure can be written in the form of a systematic large volume expansion (6.27), (6.26), in which the diagonal connected form factors of $J_{\mu}$ arise. The remarkable similarity of the large volume series of $J_{\mu}$ to the large volume series conjectures for diagonal matrix elements of local operators in purely elastic scattering theories [38-42] made us to conjecture, that formulas (6.19) and (6.28) describe the pure solitonic finite volume expectation values of any local operators of the Massive-Thirring/sine-Gordon models.

Beyond the results of this paper a lot of interesting questions are still open. It would be important to test the conjecture (6.27) and (6.28) for other operators than $J_{\mu}$. As it was demonstrated in $[51,52]$ the truncated conformal space approach could be an appropriate method for these investigations. It would be also interesting to know how the large volume series formulas (6.19), (6.28) and (6.27), (6.26) should be modified, when expectation values between not pure soliton states are considered. And finally the computation of non-diagonal finite volume form factors would be also of great importance.

Beyond the spin $+\frac{1}{2}$ light-cone lattice approach of [3], in the literature there exist other integrable lattice regularizations for the sine-Gordon model, which are based on spin $-\frac{1}{2}$ spin chains [16, 54-58]. In the framework of the spin $-\frac{1}{2}$ lattice approach of [54], local operators $[59,60]$ and their form factors $[60]$ have been computed on the lattice, but the continuum results are still missing. It would be also very interesting to see, whether this spin $-\frac{1}{2}$ approach also allows one to compute diagonal matrix elements of local operators of the continuum theory. Results of [57] could be helpful in relating the results of the two approaches, since in this paper it has been shown that the continuum equations of the spin $-\frac{1}{2}$ regularization can be mapped to those of the spin $+\frac{1}{2}$ approach.

\section{Acknowledgments}

The author would like to thank Zoltán Bajnok and János Balog for useful discussions. This work was supported by OTKA grant under K109312. The author also would like to thank the support of an MTA-Lendület Grant.

\section{A Conventions of Fourier transformation}

In this short appendix we summarize our conventions for Fourier-transformation and provide the Fourier-transform of some functions we used in section 4.1.

Our convention for the Fourier-transform of a function $f$ is given by:

$$
\tilde{f}(\omega)=\int_{-\infty}^{\infty} d x e^{i \omega x} f(x) .
$$


The inverse transformation reads as:

$$
f(x)=\int_{-\infty}^{\infty} \frac{d x}{2 \pi} e^{-i \omega x} \tilde{f}(\omega) .
$$

The Fourier-transform of the convolution two functions $f$ and $g$ is given by the product of individual Fourier-transforms:

$$
(f \star g)(x)=\int_{-\infty}^{\infty} d y f(x-y) g(y), \quad \widetilde{(f \star g)}(\omega)=\tilde{f}(\omega) \tilde{g}(\omega) .
$$

When deriving the linear equations (4.23) one needs the Fourier-transform of $K(\lambda \mid \gamma)$ of (4.12). It is given by the formula:

$$
\tilde{K}(\omega \mid \gamma)=\frac{\sinh \left[\frac{\pi \omega}{2}\left(1-\frac{2 \gamma}{\pi}\right)\right]}{\sinh \left(\frac{\pi \omega}{2}\right)} .
$$

The following inverse transform played important role at the determination of the source term in (4.23):

$$
\int_{-\infty}^{\infty} \frac{d x}{2 \pi} e^{-i \omega \lambda} \frac{\tilde{K}\left(\omega \mid \frac{\gamma}{2}\right)}{1+\tilde{K}(\omega \mid \gamma)}=\frac{1}{2 \gamma} \frac{1}{\cosh \left(\frac{\pi \lambda}{\gamma}\right)}
$$

Open Access. This article is distributed under the terms of the Creative Commons Attribution License (CC-BY 4.0), which permits any use, distribution and reproduction in any medium, provided the original author(s) and source are credited.

\section{References}

[1] Z. Bajnok, R.A. Janik and A. Wereszczyński, HHL correlators, orbit averaging and form factors, JHEP 09 (2014) 050 [arXiv: 1404.4556] [INSPIRE].

[2] G. Delfino, G. Mussardo and P. Simonetti, Nonintegrable quantum field theories as perturbations of certain integrable models, Nucl. Phys. B 473 (1996) 469 [hep-th/9603011] [INSPIRE].

[3] C. Destri and H.J. de Vega, Light-cone lattice approach to fermionic theories in 2-D: the massive thirring model, Nucl. Phys. B 290 (1987) 363 [INSPIRE].

[4] A. Klümper, M.T. Batchelor and P.A. Pearce, Central charges of the 6- and 19-vertex models with twisted boundary conditions, J. Phys. A 24 (1991) 3111 [InSPIRE].

[5] C. Destri and H.J. de Vega, New approach to thermal Bethe ansatz, hep-th/9203064 [INSPIRE].

[6] C. Destri and H.J. de Vega, Unified approach to thermodynamic Bethe Ansatz and finite size corrections for lattice models and field theories, Nucl. Phys. B 438 (1995) 413 [hep-th/9407117] [INSPIRE].

[7] D. Fioravanti, A. Mariottini, E. Quattrini and F. Ravanini, Excited state Destri-De Vega equation for sine-Gordon and restricted sine-Gordon models, Phys. Lett. B 390 (1997) 243 [hep-th/9608091] [INSPIRE]. 
[8] C. Destri and H.J. de Vega, Nonlinear integral equation and excited states scaling functions in the sine-Gordon model, Nucl. Phys. B 504 (1997) 621 [hep-th/9701107] [INSPIRE].

[9] G. Feverati, F. Ravanini and G. Takács, Nonlinear integral equation and finite volume spectrum of sine-Gordon theory, Nucl. Phys. B 540 (1999) 543 [hep-th/9805117] [InSPIRE].

[10] G. Feverati, F. Ravanini and G. Takács, Truncated conformal space at $c=1$, nonlinear integral equation and quantization rules for multi-soliton states, Phys. Lett. B 430 (1998) 264 [hep-th/9803104] [INSPIRE].

[11] G. Feverati, F. Ravanini and G. Takács, Scaling functions in the odd charge sector of sine-Gordon/massive Thirring theory, Phys. Lett. B 444 (1998) 442 [hep-th/9807160] [INSPIRE].

[12] G. Feverati, Finite volume spectrum of sine-Gordon model and its restrictions, hep-th/0001172 [INSPIRE].

[13] S.R. Coleman, The Quantum sine-Gordon Equation as the Massive Thirring Model, Phys. Rev. D 11 (1975) 2088 [inSPIRE].

[14] T.R. Klassen and E. Melzer, sine-Gordon not equal to massive Thirring and related heresies, Int. J. Mod. Phys. A 8 (1993) 4131 [hep-th/9206114] [INSPIRE].

[15] L.D. Faddeev, E.K. Sklyanin and L.A. Takhtajan, Quantum inverse problem method. 1, Theor. Math. Phys. 40 (1980) 688 [INSPIRE].

[16] V.E. Korepin, N.M. Bogoliubov and A.G. Izergin, Quantum inverse scattering method and correlation functions, Cambridge University Press, Cambridge U.K. (1993).

[17] N. Kitanine, J.M. Maillet and V. Terras, Form factors of the XXZ Heisenberg spin-1/2 finite chain, Nucl. Phys. B 554 (1999) 647 [math-ph/9807020].

[18] N. Kitanine, J.M. Maillet and V. Terras, Correlation functions of the XXZ Heisenberg spin-1/2 chain in a magnetic field Nucl. Phys. B 567 (2000) 554 [math-ph/9907019].

[19] N. Kitanine, J.M. Maillet, N.A. Slavnov and V. Terras, Spin spin correlation functions of the XXZ-1/2 Heisenberg chain in a magnetic field, Nucl. Phys. B 641 (2002) 487 [hep-th/0201045] [INSPIRE].

[20] N. Kitanine, J.M. Maillet, N.A. Slavnov and V. Terras, Dynamical correlation functions of the XXZ spin-1/2 chain, Nucl. Phys. B 729 (2005) 558 [hep-th/0407108] [INSPIRE].

[21] J.M. Maillet and V. Terras, On the quantum inverse scattering problem, Nucl. Phys. B 575 (2000) 627 [hep-th/9911030] [INSPIRE].

[22] A.G. Izergin, N. Kitanine, J.M. Maillet and V. Terras, Spontaneous magnetization of the XXZ Heisenberg spin-1/2 chain, Nucl. Phys. 554 (1999) 679 [solv-int/9812021].

[23] N. Kitanine, J.M. Maillet, N.A. Slavnov and V. Terras, Master equation for spin-spin correlation functions of the XXZ chain, Nucl. Phys. B 712 (2005) 600 [hep-th/0406190] [INSPIRE].

[24] N. Kitanine, K.K. Kozlowski, J.M. Maillet, N.A. Slavnov and V. Terras, Algebraic Bethe ansatz approach to the asymptotic behavior of correlation functions, J. Stat. Mech. 0904 (2009) P04003 [arXiv:0808.0227] [INSPIRE].

[25] N. Kitanine, K.K. Kozlowski, J.M. Maillet, N.A. Slavnov and V. Terras, On the thermodynamic limit of form factors in the massless XXZ Heisenberg chain, J. Math. Phys. 50 (2009) 095209 [arXiv:0903.2916] [inSPIRE]. 
[26] N. Kitanine, K.K. Kozlowski, J.M. Maillet, N.A. Slavnov and V. Terras, Form factor approach to the asymptotic behavior of correlation functions in critical models,

J. Stat. Mech. 1112 (2011) P12010 [arXiv:1110.0803] [InSPIRE].

[27] N. Kitanine, K.K. Kozlowski, J.M. Maillet, N.A. Slavnov and V. Terras, The thermodynamic limit of particle-hole form factors in the massless XXZ Heisenberg chain,

J. Stat. Mech. 1105 (2011) P05028 [arXiv:1003.4557] [INSPIRE].

[28] N. Kitanine, K.K. Kozlowski, J.M. Maillet and V. Terras, Large-distance asymptotic behaviour of multi-point correlation functions in massless quantum models, J. Stat. Mech. 1405 (2014) P05011 [arXiv:1312.5089] [INSPIRE].

[29] N. Kitanine, K.K. Kozlowski, J.M. Maillet, N.A. Slavnov and V. Terras, Form factor approach to dynamical correlation functions in critical models, J. Stat. Mech. 1209 (2012) P09001 [arXiv:1206.2630] [INSPIRE].

[30] M. Dugave, F. Göhmann and K.K. Kozlowski, Thermal form factors of the XXZ chain and the large-distance asymptotics of its temperature dependent correlation functions, J. Stat. Mech. (2013) P07010 [arXiv: 1305. 0118] [INSPIRE].

[31] M. Dugave, F. Göhmann and K.K. Kozlowski, Low-temperature large-distance asymptotics of the transversal two-point functions of the XXZ chain, J. Stat. Mech. 1404 (2014) P04012 [arXiv: 1401.4132] [INSPIRE].

[32] M. Dugave, F. Göhmann, K.K. Kozlowski and J. Suzuki, Thermal form factor approach to the ground-state correlation functions of the $X X Z$ chain in the antiferromagnetic massive regime, J. Phys. A 49 (2016) 394001 [arXiv:1605.07968] [INSPIRE].

[33] J.S. Caux, R. Hagemans and J.M. Maillet, Computation of dynamical correlation functions of Heisenberg chains: the gapless anisotropic regime, J. Stat. Mech. 9 (2005) P09003 [cond-mat/0506698].

[34] J.-S. Caux and J.-M. Maillet, Computation of dynamical correlation functions of Heisenberg chains in a field, Phys. Rev. Lett. 95 (2005) 077201 [cond-mat/0502365] [INSPIRE].

[35] A.G. Izergin, Partition function of the six-vertex model in a finite volume, Sov. Phys. Dokl. 32 (1987) 878.

[36] N.A. Slavnov, Calculation of scalar products of wave functions and form factors in the framework of the algebraic Bethe Ansatz, Theor. Math. Phys. 79 (1989) 502.

[37] S.L. Lukyanov and V. Terras, Long distance asymptotics of spin spin correlation functions for the XXZ spin chain, Nucl. Phys. B 654 (2003) 323 [hep-th/0206093] [INSPIRE].

[38] A. Leclair and G. Mussardo, Finite temperature correlation functions in integrable QFT, Nucl. Phys. B 552 (1999) 624 [hep-th/9902075] [INSPIRE].

[39] H. Saleur, A Comment on finite temperature correlations in integrable QFT, Nucl. Phys. B 567 (2000) 602 [hep-th/9909019] [INSPIRE].

[40] B. Pozsgay, Mean values of local operators in highly excited Bethe states, J. Stat. Mech. 1101 (2011) P01011 [arXiv: 1009.4662] [INSPIRE].

[41] B. Pozsgay, Form factor approach to diagonal finite volume matrix elements in Integrable QFT, JHEP 07 (2013) 157 [arXiv: 1305.3373] [INSPIRE].

[42] B. Pozsgay, I.M. Szécsényi and G. Takács, Exact finite volume expectation values of local operators in excited states, JHEP 04 (2015) 023 [arXiv: 1412.8436] [INSPIRE]. 
[43] T. Pálmai and G. Takács, Diagonal multisoliton matrix elements in finite volume, Phys. Rev. D 87 (2013) 045010 [arXiv: 1209.6034] [inSPIRE].

[44] J.S. Maybee, D.D. Olesky, P. van den Driessche and G. Wiener, Matrices, digraphs, and determinants, SIAM J. Matrix Anal. Appl. 10 (1989) 500.

[45] M. Fowler and X. Zotos, Bethe-ansatz quantum sine-Gordon thermodynamics. The specific heat, Phys. Rev. B 25 (1982) 5806.

[46] J. Balog and A. Hegedüs, TBA equations for excited states in the sine-Gordon model, J. Phys. A 37 (2004) 1903 [hep-th/0304260] [INSPIRE].

[47] B. Pozsgay and G. Takács, Form-factors in finite volume I: Form-factor bootstrap and truncated conformal space, Nucl. Phys. B 788 (2008) 167 [arXiv:0706.1445] [INSPIRE].

[48] B. Pozsgay and G. Takács, Form factors in finite volume. II. Disconnected terms and finite temperature correlators, Nucl. Phys. B $\mathbf{7 8 8}$ (2008) 209 [arXiv:0706.3605] [INSPIRE].

[49] M. Jimbo, T. Miwa and F. Smirnov, Hidden Grassmann structure in the XXZ model V: sine-Gordon model, Lett. Math. Phys. 96 (2011) 325 [arXiv:1007.0556] [InSPIRE].

[50] M. Jimbo, T. Miwa and F. Smirnov, On one-point functions of descendants in sine-Gordon model, arXiv:0912.0934 [INSPIRE].

[51] G. Fehér and G. Takács, sine-Gordon form factors in finite volume, Nucl. Phys. B 852 (2011) 441 [arXiv:1106.1901] [INSPIRE].

[52] G.Z. Fehér, T. Pálmai and G. Takács, sine-Gordon multi-soliton form factors in finite volume, Phys. Rev. D 85 (2012) 085005 [arXiv: 1112.6322] [InSPIRE].

[53] F. Buccheri and G. Takács, Finite temperature one-point functions in non-diagonal integrable field theories: the sine-Gordon model, JHEP 03 (2014) 026 [arXiv:1312.2623] [INSPIRE].

[54] A.G. Izergin and V.E. Korepin, The lattice quantum sine-Gordon model, Lett. Math. Phys. 5 (1981) 199 [INSPIRE].

[55] D. Fioravanti and M. Rossi, A Braided Yang-Baxter algebra in a theory of two coupled lattice quantum KdV: Algebraic properties and ABA representations, J. Phys. A 35 (2002) 3647 [hep-th/0104002] [INSPIRE].

[56] D. Fioravanti and M. Rossi, From the braided to the usual Yang-Baxter relation, J. Phys. A 34 (2001) L567 [hep-th/0107050] [INSPIRE].

[57] D. Fioravanti and M. Rossi, Exact conserved quantities on the cylinder 1: Conformal case, JHEP 07 (2003) 031 [hep-th/0211094] [INSPIRE].

[58] D. Fioravanti and M. Rossi, Exact conserved quantities on the cylinder. 2. Off critical case, JHEP 08 (2003) 042 [hep-th/0302220] [INSPIRE].

[59] T. Oota, Quantum projectors and local operators in lattice integrable models, J. Phys. A 37 (2004) 441 [hep-th/0304205] [inSPIRE].

[60] N. Grosjean, J.M. Maillet and G. Niccoli, On the form factors of local operators in the lattice sine-Gordon model, J. Stat. Mech. 10 (2012) 006 [arXiv:1204.6307] [INSPIRE]. 